Copyright

by

Romain Fiévet

2014 
The Thesis commitee of Romain Fiévet

certifies that this is the approved version of the following thesis:

\title{
Acoustic waveforms produced by a laboratory scale supersonic jet
}

\author{
APPROVED BY \\ SUPERVISING COMMITTEE:
}

Charles E. Tinney, Supervisor

Mark F. Hamilton 


\title{
Acoustic waveforms produced by a laboratory scale supersonic jet
}

by

\section{Romain Fiévet}

\author{
THESIS \\ Presented to the Faculty of the Graduate School of \\ The University of Texas at Austin \\ in Partial Fulfillment \\ of the Requirements \\ for the Degree of
}

Master of Science in Engineering

THE UNIVERSITY OF TEXAS AT AUSTIN

August 2014 
Dedicated to my family. 


\section{Acknowledgments}

I wish to first thank my advisor Dr.Charles Tinney who offered me the opportunity to join the Aerospace graduate program at UT, for his guidance during these past two-years, and the many insightful conversations we had. What I learnt under his supervision will be extremely precious during my future career. I also wish to particularly thank Dr.Woutijn Baars for his patience, for all the advice he graciously gave me during my first year in the group, and for his continuous involvement in this project. I would also like to thank Dr.Mark Hamilton for his very valuable collaboration. Finally, I thank my close friends and my family for their continuous support.

This work was funded through the Office of Naval Research Jet Noise Reduction program, contract number N00014-11-1-0752, under the direction of Drs. Joseph Doychak and Brenda Henderson. 


\title{
Acoustic waveforms produced by a laboratory scale supersonic jet
}

\author{
Romain Fiévet, M.S.E. \\ The University of Texas at Austin, 2014
}

Supervisor: Charles E. Tinney

The spatial evolution of acoustic waveforms produced by a Mach 3 jet are investigated using both $1 / 4$ inch and 1/8 inch pressure field microphones located along rays emanating from the post potential core where the peak sound emission is found to occur. The measurements are acquired in a fully anechoic chamber where ground, or other large surface reflections are minimal. The calculation of the OASPL along an arc located at 95 jet diameters using 120 planar grid measurements are shown to collapse remarkably well when the arc array is centered on the post potential core region. Various statistical metrics, including the quadrature spectral density, number of zero crossings, the skewness of the pressure time derivative and the integral of the negative part of the quadrature spectral density, are exercised along the peak emission path. These metrics are shown to undergo rapid changes within 2 meters from the source regions of this laboratory scale jet. The sensitivity of these findings to both transducer size and humidity effects are discussed. A visual extrapolation of these nonlinear metrics toward the jet shear layer suggests that these waveforms are initially skewed at the source. An experimentally validated 
wave packet model is used to confirm the location where the pressure decay law transition from cylindrical to spherical. It is then used to estimate the source intensity, which is required to predict the effective Gol'dberg number. 


\section{Nomenclature}

$A$ non-dimensional wave packet parameter, $L / D_{j}$

$a_{j} \quad$ speed of sound at the jet nozzle exit, $\mathrm{m} / \mathrm{s}$

$a_{\infty} \quad$ ambient speed of sound, $\mathrm{m} / \mathrm{s}$

$D_{j} \quad$ jet exit diameter, $\mathrm{m}$

$f_{c} \quad$ cut-off frequency, $\mathrm{Hz}$

$f_{j} \quad$ jet characteristic frequency, $\mathrm{Hz}$

$f_{0} \quad$ peak spectral frequency along the propagation path, $\mathrm{Hz}$

$G_{p p} \quad$ pressure one-sided power spectral density, $\mathrm{Pa}^{2} / \mathrm{Hz}$

L wave packet model scale

$\ell_{\alpha} \quad$ absorption length scale

$M_{a} \quad$ acoustic Mach number, $U_{j} / a_{\infty}$

$M_{j} \quad$ jet exit Mach number, $U_{j} / a_{j}$

$M_{c} \quad$ Convective Mach number, $U_{c} / a_{\infty}$

$N \quad$ Number of microphones

$N_{z c} \quad$ Number of zero crossings, \#/s

$p \quad$ pressure waveform, $\mathrm{Pa}$

$\dot{p} \quad$ time derivative of the pressure waveform, $\mathrm{Pa} / \mathrm{s}$

$\hat{p} \quad$ Fourier transform of the time pressure signal, $\mathrm{Pa} / \sqrt{\mathrm{Hz}}$ 
$P_{0} \quad$ total pressure at the jet nozzle exit, $\mathrm{K}$

$P_{\infty} \quad$ ambient pressure, $\mathrm{K}$

$Q \quad$ normalized quadrature spectral density, $1 / \mathrm{Hz}$

$Q_{n e g}$ integral of the negative part of $Q$

$Q_{p^{2} p} \quad$ quadrature spectral density of the pressure, $\mathrm{Pa}^{3} / \mathrm{Hz}$

$r$ radial coordinate relative to the jet centerline.

$\bar{r}_{c} \quad$ cylindrical shock formation distance, $\mathrm{m}$

$\bar{r}_{s} \quad$ spherical shock formation distance, $\mathrm{m}$

$\%$ RH percent relative humidity

$S_{p p} \quad$ pressure two-sided power spectral density, $\mathrm{Pa}^{2} / \mathrm{Hz}$

$S t_{D_{j}} \quad$ Strouhal number: $f D_{j} / U_{j}$

$t \quad$ time, $\mathrm{s}$

$T_{0} \quad$ total temperature at the jet nozzle exit, $\mathrm{K}$

$T_{j} \quad$ static temperature at the jet nozzle exit, $\mathrm{K}$

$T_{\infty} \quad$ ambient temperature, $\mathrm{K}$

$U_{c} \quad$ convective speed of the jet, $\mathrm{m} / \mathrm{s}$

$U_{j} \quad$ velocity at the jet nozzle exit, $\mathrm{m} / \mathrm{s}$

$w \quad$ wave packet amplitude

$W_{\text {error }}$ Wave packet error

$x \quad$ axial coordinate relative to the jet exit plane

$\bar{x} \quad$ plane wave shock formation distance

$\alpha \quad$ absorption factor, $\mathrm{Np} / \mathrm{m}$ 
$\beta \quad$ coefficient of nonlinearity

$\gamma \quad$ skewness factor

$\Gamma \quad$ Gol'dberg number for plane waves

$\kappa \quad$ kurtosis factor

$\Lambda_{c} \quad$ modified cylindrical Gol'dberg number

$\Lambda_{s} \quad$ modified spherical Gol'dberg number

$\phi \quad$ Mach wave angle measured from the jet centerline, deg.

$\rho \quad$ distance along a microphone array, $\mathrm{m}$

$\rho_{\infty} \quad$ ambient density, $\mathrm{kg} / \mathrm{m}^{3}$

$\sigma_{p, 0} \quad$ pressure source strength, $\mathrm{Pa}$

$\sigma_{p} \quad$ standard deviation of $p(t)$

$\theta \quad$ angle measured from the jet centerline, deg.

$\tau \quad$ retarded time, $\mathrm{s}$

$\xi_{p p} \quad$ pressure cross correlation, $\mathrm{Pa}^{2}$

FSS Fine Scale Similarity spectra

LSS Large Scale Similarity spectra

NPR Nozzle pressure ratio, $P_{0} / P_{\infty}$

OASPL Overall sound pressure level, dB, ref:20 $\mu \mathrm{Pa}$

PDF Probability density function

SPL Sound pressure level, dB, ref: $(20 \mu \mathrm{Pa})^{2} / \mathrm{Hz}$ 


\section{Table of Contents}

Acknowledgments $\quad$ v

Abstract vi

Nomenclature viii

List of Tables $\quad$ xiii

List of Figures xiv

$\begin{array}{lll}\text { Chapter 1. Introduction } & 1\end{array}$

Chapter 2. Experimental Set-up 4

2.1 Facility overview . . . . . . . . . . . . . . . . 4

2.2 Jet operating conditions . . . . . . . . . . . . . 5

2.3 Instrumentation and microphone placement . . . . . . . . . . . 9

$\begin{array}{lll}\text { Chapter 3. Experimental results } & 14\end{array}$

3.1 Preliminary corrections and spectral observations . . . . . . . 14

3.2 Sound pressure decay in the jet far-field . . . . . . . . . . . . . 17

3.3 Wave packet .................. 22

Chapter 4. Analysis of acoustic waveforms along the peak emission path $\quad 27$

4.1 Spectral indicators . . . . . . . . . . . . . . . 30

4.2 Scalar indicators . . . . . . . . . . . . . . . . . 31

4.3 Shock formation distance . . . . . . . . . . . . . . . . . . 35

4.4 Gol'dberg number . . . . . . . . . . . . . . . . . . 37

4.5 Direct observation . . . . . . . . . . . . . . . 38 
Chapter 5. Conclusions

Bibliography 


\section{List of Tables}

2.1 Testing conditions for the 1/8 inch microphone study. Average values from the $1 / 4$ inch study are shown in parenthesis. Note: the microphone locations corresponding to the $45_{1}^{\circ}$ and $45_{2}^{\circ}$ arrays are provided in Table $2.2 . \ldots . . . . . .10$

2.2 Locations of $1 / 8$ inch microphones. . . . . . . . . . . . . . . . 13

4.1 Shock formation distances and effective Gol'dberg numbers. . 37 


\section{List of Figures}

2.1 The fully anechoic chamber at UT Austin during the current set of measurements. . . . . . . . . . . . . . .

2.2 Normal incidence absorption coefficient of a UT Austin chamber wedge with $10 \mathrm{~cm}$ air cavity. . . . . . . . . . . .

2.3 Evolution of the NPR and the valve opening \% through a typical test session. . . . . . . . . . . . . . .

2.4 Block diagram presenting the different phases of the whole experimental process. . . . . . . . . . . .

2.5 Location of $1 / 8$ inch microphone rays and coordinate system. Contour of OASPL $(\mathrm{dB}$, ref: $20 \mu \mathrm{Pa})$ from $1 / 4$ inch $(x, r)$ grid measurements of Baars \& Tinney (2014). . . . . . . . . . . .

3.1 (a) One-sided power spectral density (PSD) with and without the $1^{\text {st }} / 2^{\text {nd }}$ order high-pass filter correction. (b) PSD of a sample microphone signal at $45^{\circ}$ with and without corrections using manufacturer supplied data. . . . . . . . . . . . . .

3.2 Spectral comparison with $4^{\text {th }}$ order Butterworth and grid caps off/off (red), off/on (dark gray) and on/on (black) for a microphone in the middle of the array $\left(\mathrm{x}=75 D_{j}\right)$; the light gray line shows the difference the filter causes to the spectrum. . . . . .

3.3 PSD at $\theta=35^{\circ}$ and $65^{\circ}$ being compared to the LSS and FSS of Tam et al. (1996). . . . . . . . . . . . . . . . . . .

3.4 (a) PSD for two different testing sessions (black/red) with identical experimental conditions at all microphone positions along an array (spectra shifted by $10 \mathrm{~dB}$ ). (b) Evolution of the PSD along the second $45^{\circ}$ array (blue for the first microphone, black for the last one). . . . . . . . . . . . . . . .

3.5 (a) $\sigma_{p}$ decay and (b) $\sigma_{p}^{-1} \times 10^{2}$ from $1 / 8$ inch microphones. . .

3.6 OASPL (dB, ref: $20 \mu \mathrm{Pa})$ from $(x, r)$ plane grid measurements of Baars \& Tinney (2014) projected to an arc array at $\rho / D_{j}=95$ using spherical decay. Assuming source region at (a) $x / D_{j}=0$ and (b) $x / D_{j}=17.5$. The values shown are less $100 \mathrm{~dB} . .$. 
3.7 (a) Schematic of the wave packet scale versus jet scale. (b) Regions where the wave packet model obeys cylindrical and spherical spreading along the peak noise path. Contour lines define error thresholds of $2 \%, 3 \%$ and $4 \%$. . . . . . .

3.8 Integrated errors between the wave packet model and laboratory measurements for various wave packet length scales and source locations along the propagation path. Contour levels have been normalized so that they range between 0 and 1 with increments of $0.125 \ldots \ldots \ldots \ldots \ldots \ldots \ldots$

3.9 Spreading trends along the peak noise emission path obtained from the wave packet model using $A=8.75$. Both experimental datasets are shown with $\square$ corresponding to the $1 / 4$ inch microphones. . . . . . . . . . . . .

4.1 (a) $Q \times 10^{-5}$ and (b) $Q / S$, Morfey-Howell indicators (1\% bandwidth moving filter) for all microphones (gray) and for the last 4 microphones (black). . . . . . . . . . . . . . .

4.2 (a) $Q$ and (b) $Q / S$ with and without the $1 \%$ bandwidth moving filter $(\mathrm{BMF}) \ldots \ldots \ldots \ldots \ldots \ldots \ldots \ldots$

4.3 (a) $N_{z c}$, (b) $\gamma(\dot{p}),\left(\right.$ c) $\kappa(\dot{p})$, (d) $-Q_{n e g} \times 10^{2}$ along the $45^{\circ}$ ray and (e) comparison of their normalized spatial derivatives. . .

4.4 Evolution of the raw pressure time series (shown as $p(\rho, t) / \sigma_{p}(\rho)$ ) along the $45_{1}^{\circ}$ ray in the inner zone encompassing $\rho / D_{j}=[13$ : $5: 48] \ldots \ldots \ldots \ldots \ldots \ldots \ldots \ldots$

4.5 Evolution of the raw pressure time series (shown as $p(\rho, t) / \sigma_{p}(\rho)$ ) along the $45_{2}^{\circ}$ ray in the outer zone encompassing $\rho / D_{j}=[35.5$ : $10: 105.5] \ldots \ldots \ldots \ldots \ldots \ldots \ldots$

4.6 Cross-correlations between microphones on the $45_{2}^{\circ}$ ray in the outer zone encompassing $\rho / D_{j}=[35.5: 10: 105.5]$. . . . . . 42

4.7 (a) A rogue wave and (b) a passing wave. . . . . . . . . . 43 


\section{Chapter 1}

\section{Introduction}

When the convective speed of large scale turbulent structures is greater than the sound speed of the surrounding gas, Mach waves are emitted which propagate at angles relative to the jet axis determined by $\phi=\cos ^{-1}\left(a_{\infty} / U_{c}\right)[4,9,10,22,27,36]$. Because these Mach waves are generated by turbulence, they are classified as a component of turbulent mixing noise, which is the most significant source of noise for jets operating at supersonic acoustic Mach numbers. If the amplitude of the Mach wave is large enough to overcome viscous relaxation effects $[7,15]$, the waveform will steepen with distance from its source, which is more easily observed under full-scale conditions where the characteristic frequencies, and hence viscous relaxation effects, are low [12].

While sawtooth-like waveforms have been observed in both laboratory and full scale jet noise studies, the source and nature of these shapes remains unknown. Most laboratory and full-scale studies are conducted using arc arrays of microphones placed in the far-field of the jet (beyond 60 or so jet diameters). And so the shape and evolution of these Mach waves, as they propagate from the source regions of the flow to the far-field, are not well understood. A recent study by Baars, Tinney \& Wochner (2012) [2] mapped the acoustic field of an unheated supersonic jet (between $x / D_{j}=[5: 10: 145]$ and $\left.r / D_{j}=[25: 10: 95]\right)$ operating at a gas dynamic Mach number of 3 to unveil 
the sound field produced by jets comprising supersonic acoustic Mach numbers. Baars \& Tinney (2014) [4] later postulated that steepened waveforms are produced either at the source or develop rapidly in the near-field through cumulative nonlinear distortion effects (much earlier than where typical farfield measurements are acquired). Recent Large-Eddy-Simulations by Nichols et al. (2013) [30] showed sawtooth waves being emitted from within the jet shear layer and that, despite further steepening from cumulative nonlinear distortions, it was likely that steepened waveforms were also being generated within the jet. Anderson \& Freund (2012) [1] observed similar features using direct numerical simulations and went on to illustrate the clumping together of shorter waves in the very near field of supersonic mixing layers. Such observations would suggest that large amplitude waves do not form on their own, but rather form by partnering with other smaller waves to produce larger and more steepened waves further from the jet. Thus, the observations of Nichols et al. (2013) [30], Baars \& Tinney (2014) [4] and Anderson \& Freund (2012) [1] suggest that the emulsion of skewed waveforms, from within the jet, proceeded by either rapid waveform steepening or the partnering of similar waves along the propagation path are responsible for sawtooth like structures observed in the far-field. And so, an important question that we seek to address here is whether the acoustic waveforms, produced by Mach waves emanating from laboratory scale jets, undergo cumulative nonlinear distortions, or steepen through other mechanisms.

Here, we will replicate portions of the experiment performed by Baars et al. (2012) [2], who used 1/4 inch microphones to characterize the acoustic field of a Mach 3 jet. Contrary to that study, we will employ higher fidelity instruments (1/8 inch pressure field microphones), positioned closer to the jet 
flow and along lines emanating from the post potential core region where the prominent source of sound is known to reside. As was done before, various well known statistical metrics for quantifying waveform steepening and coalescence will be exercised to determine the shape and nature of the waveforms as they propagate along rays centered on the post-potential core. The effect of absorption on these various metrics will be studied, as the measurements of Baars et al. (2012) [2] were conducted at higher relative humidities than the measurements acquired with the $1 / 8$ inch microphones. Doing so will increase the likelihood of capturing, over a broader spectral range, the acoustic waves as they radiate to the far-field of laboratory scale supersonic jets. 


\section{Chapter 2}

\section{Experimental Set-up}

\subsection{Facility overview}

Both the experiments of Baars et al. (2012) [2] and the current higher fidelity measurements were conducted in the fully anechoic chamber and open jet wind tunnel at The University of Texas at Austin. An illustration of the chamber is shown in Figure 2.1 and comprises internal dimensions (wedge-tip to wedge-tip) of $5.74 \mathrm{~m}($ length $) \times 4.52 \mathrm{~m}$ (width $) \times 3.66 \mathrm{~m}$ (height). The acoustic wedges that are used to produce this echo free environment are composed of $46 \mathrm{~cm}$ of fire retardant melamine foam backed by a $10 \mathrm{~cm}$ air cavity followed by $14 \mathrm{~cm}$ of recycled cotton fibre. The findings from impedance tube tests performed at ETS Lindgren (Cedar Park, Texas) are shown in Figure 2.2 to demonstrate how this particular wedge design produces a normal incidence absorption coefficient of $99 \%$ above $100 \mathrm{~Hz}$ for an air cavity depth of $10 \mathrm{~cm}$; the small dip around $250 \mathrm{~Hz}$ is due to the flow resistive properties of the melamine foam.

A jet rig is positioned along the centerline of the chamber and tunnel, as shown in Figure 2.1 with a $1.49 \mathrm{~m}^{2}$ square aperture on the back wall to allow entrained flow to enter into the chamber. Both the jet and entrained flows exhaust downstream through a $1.83 \mathrm{~m}^{2}$ acoustically treated square duct and with the assistance of a vane axial fan to reduce recirculations from forming inside the chamber. The nozzle tested is the same as that described by Baars 
et al. (2012) [2] and was designed to produce a perfectly expanded flow at a jet exit Mach number of 3.0 with an exit diameter of $D_{j}=0.0254 \mathrm{~m}$ using unheated air. Nozzle pressure ratios (NPR) are monitored and controlled using a National Instruments CompactRIO system which is capable of reducing variations in the set-point NPR to less than $1 \%$ over extended periods of time using a proportional-integral-derivative (PID) controller. This high level of accuracy in the nozzle test conditions is achieved by operating two valves in parallel. The first valve passes the bulk fluid and is operated manually in an open loop setting. A second smaller tuning valve is then operated by way of the PID controller. Figure 2.3 illustrates the percentage opening of the primary and secondary tuning valves during a typical test. Likewise, a process and instrumentation diagram is provided in Figure 2.4. Additional details concerning this facility can be found in Donald et al. (2014) [8] and Baars (2013) [3]. The overall experimental process (jet operating conditions, data acquisition and post-processing) is detailed in the block diagram 2.4.

\subsection{Jet operating conditions}

Five separate tests were conducted in September 2013 over a duration of 2 days during which atmospheric conditions were carefully recorded; see Table 2.1. While the fully anechoic chamber and wind tunnel are located in a large warehouse; outside air is drawn in to prevent the building from collapsing during runs. Therefore, the environment inside the chamber is that of the air outside of the building. The nozzle was operated at a nozzle pressure ratio of 36.7 which was expected to produce a perfectly expanded flow at the exit of the nozzle (based on quasi 1-D isentropic compressible flow equations). Shadowgraph images later revealed the presence of weak shock structures at this 


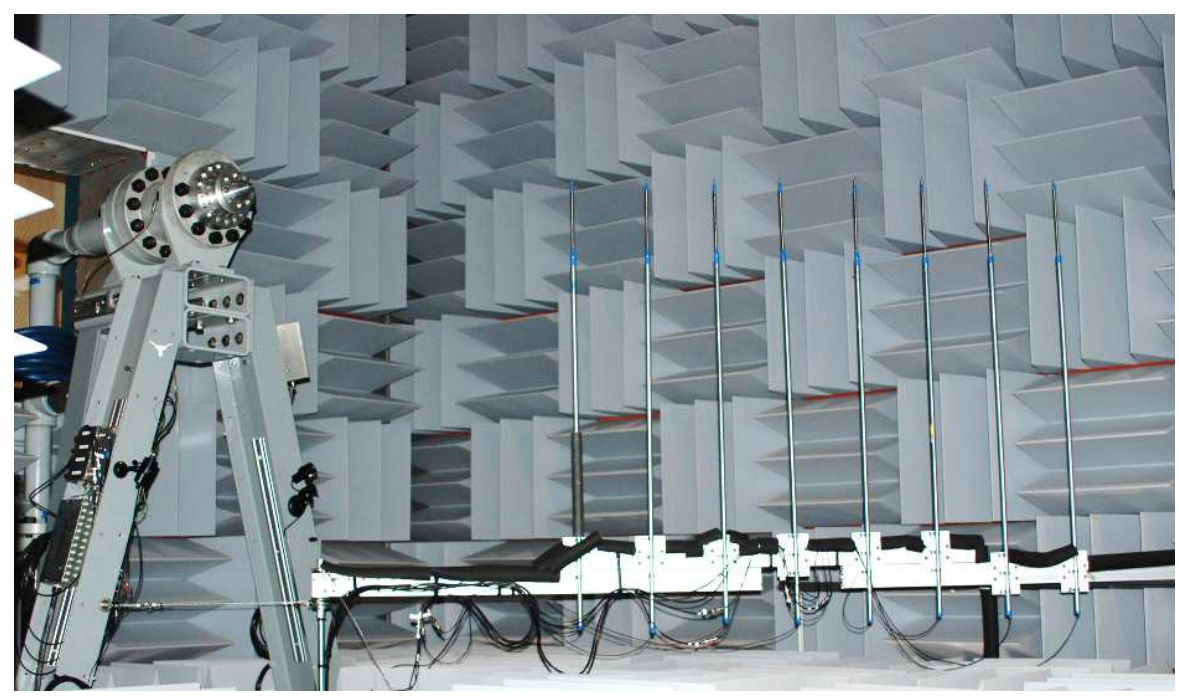

Figure 2.1: The fully anechoic chamber at UT Austin during the current set of measurements.

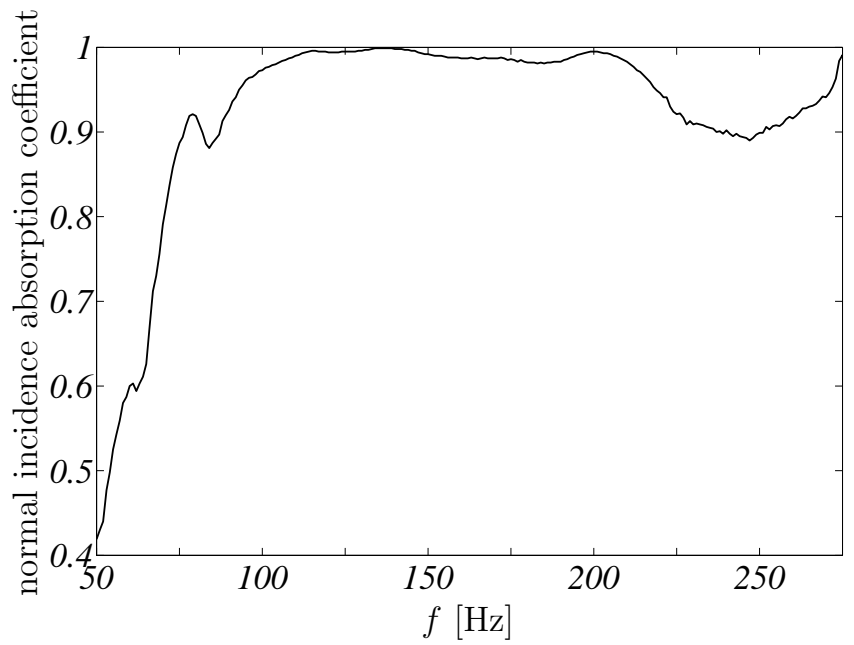

Figure 2.2: Normal incidence absorption coefficient of a UT Austin chamber wedge with $10 \mathrm{~cm}$ air cavity. 


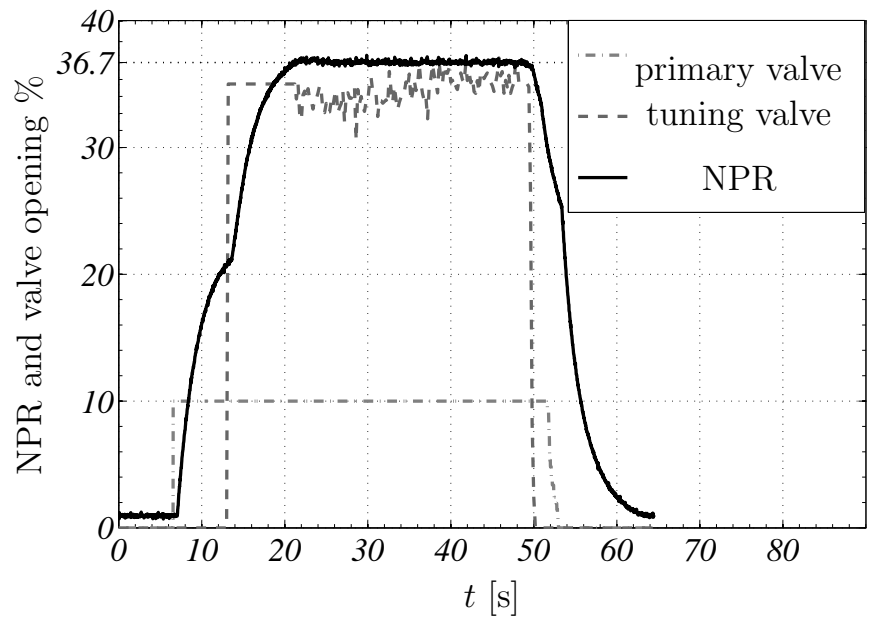

Figure 2.3: Evolution of the NPR and the valve opening \% through a typical test session.

pressure ratio. However, no broadband shock tones or screech were observed in any of the acoustic spectra, and so these shocks were not expected to have any influence on the measurements reported here (or those acquired in 2011 with the $1 / 4$ inch microphones [2-4]). A common set of values for $P_{\infty}, T_{\infty}$ and $T_{0}$ were chosen by averaging over the test conditions reported in Table 2.1. The resultant differential error between these tests was found to be within $1.5 \%$ of the average value. Included in this Table are the average values reported by Baars et al. (2012) [2], (shown in parenthesis) which compare reasonably well to the conditions reported here. The largest discrepancy between the current set of measurements and the measurements of Baars et al. (2012) [2] reside in the atmospheric conditions (relative humidity and atmospheric temperature), with percent relative humidities of 75.5, 63.2 and 48.8 being recorded by Baars et al. (2012) [2] on the three days of testing, respectively.

The convective speed of the sound producing events is an important 


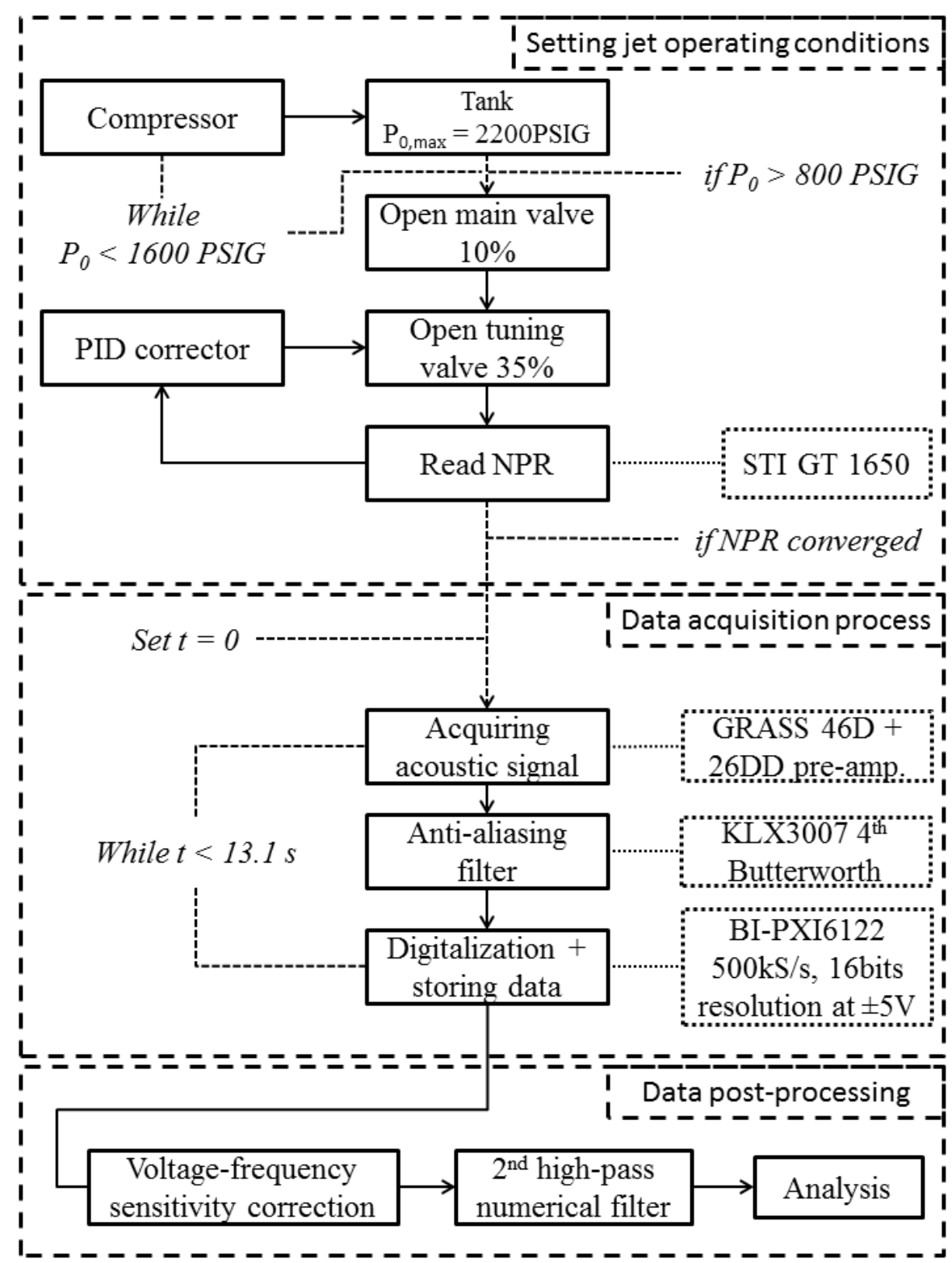

Figure 2.4: Block diagram presenting the different phases of the whole experimental process. 
quantity and is assumed to be $0.8 U_{j}$ [27]. This results in Mach wave angles around $\phi_{0.8}=45^{\circ}$, which coincide quite well with the peak noise emission angle shown in Figure 2.5; this angle is determined to be where the overall sound pressure level (OASPL) contour, also shown in Figure 2.5 from the $(x, r)$ grid measurements of Baars et al. (2012) [2], is maximum. The lobe structure in the OASPL spatial distribution is manifest and inherent to jet noise, its shape being determined by the competing effects of convection and refraction. However, closer inspection of the OASPL ridge demonstrates how contour levels, residing closer to the jet, indicate Mach waves with angles $(\phi)$ coinciding with convective speeds on the order of $0.7 U_{j}$ (identified in Figure 2.5 by $\left.\phi_{0.7}=35^{\circ}\right)$. Contrarily, in regions further from the jet, Mach wave angles coincide with structures moving at $0.8 U_{j}$ (identified in Figure 2.5 by $\phi_{0.8}=$ $45^{\circ}$ ). Variations in the Mach wave ridge are attributed to differences in the convective speed of the more pronounced sound producing events and their locations in the post potential core region relative to one another.

\subsection{Instrumentation and microphone placement}

Because results from two different studies employing different kinds of instruments are being evaluated, the details concerning both are provided here for completeness. The first of the acoustic measurements is described by Baars et al. (2012)[2] and was performed in December of 2011 using four 1/4 inch prepolarized, pressure-field condenser microphones (PCB model 377B10 capsules with 426B03 preamplifiers) positioned at grazing incidence to the jet axis. These IEPE type transducers were powered using a NI PXI-4472 card which then digitized the signals at $102.4 \mathrm{kS} / \mathrm{s}$ with 24 bit resolution $( \pm 10 \mathrm{v})$ for a minimum of $2^{20}$ samples. Signal attenuation occurs at 0.84 times the 
Table 2.1: Testing conditions for the $1 / 8$ inch microphone study. Average values from the $1 / 4$ inch study are shown in parenthesis. Note: the microphone locations corresponding to the $45_{1}^{\circ}$ and $45_{2}^{\circ}$ arrays are provided in Table 2.2.

\begin{tabular}{|c|c|c|c|c|c|c|}
\hline \multirow[b]{2}{*}{ Array angle } & \multirow{2}{*}{$\frac{\text { Measured }}{35^{\circ}}$} & \multirow[b]{2}{*}{$45_{1}^{\circ}$} & \multirow[b]{2}{*}{$45_{2}^{\circ}$} & \multirow[b]{2}{*}{$52^{\circ}$} & \multirow[b]{2}{*}{$65^{\circ}$} & \multirow[b]{2}{*}{ Average } \\
\hline & & & & & & \\
\hline$P_{\infty}, \mathrm{kPa}$ & 100.0 & 100.1 & 100.0 & 100.2 & 100.2 & $100.1(100.9)$ \\
\hline$P_{0} / P_{\infty}$ & \multicolumn{5}{|c|}{$36.7 \pm 1 \%( \pm 4.5 \%)$} & 36.7 \\
\hline$T_{\infty}, \mathrm{K}$ & 302 & 304 & 306 & 303 & 303 & $304(289)$ \\
\hline$T_{0}, \mathrm{~K}$ & 301 & 298 & 301 & 304 & 304 & $302(288)$ \\
\hline \multirow[t]{2}{*}{$\% \mathrm{RH}$} & 43 & 51 & 40 & 56 & 58 & $\mathrm{~N} / \mathrm{A}$ \\
\hline & Computed & & & & & \\
\hline$T_{j}, \mathrm{~K}$ & 108 & 106 & 108 & 109 & 109 & $108(103)$ \\
\hline$a_{j}, \mathrm{~m} / \mathrm{s}$ & 208 & 207 & 208 & 209 & 209 & $208(203)$ \\
\hline$U_{j}, \mathrm{~m} / \mathrm{s}$ & 624 & 620 & 624 & 627 & 627 & $624(609)$ \\
\hline$f_{j}, \mathrm{kHz}$ & 24.6 & 24.4 & 24.6 & 24.7 & 24.7 & $24.6(24.0)$ \\
\hline$M_{j}=U_{j} / a_{j}$ & 3.0 & 3.0 & 3.0 & 3.0 & 3.0 & 3.0 \\
\hline$M_{a}=U_{j} / a_{\infty}$ & 1.78 & 1.78 & 1.78 & 1.80 & 1.80 & $1.79(1.79)$ \\
\hline$M_{c}=0.8 U_{j} / a_{\infty}$ & 1.43 & 1.42 & 1.42 & 1.44 & 1.44 & $1.43(1.43)$ \\
\hline$\phi_{0.8}$, deg. & 45 & 45 & 45 & 46 & 46 & $45(46)$ \\
\hline$\phi_{0.7}$, deg. & 37 & 36 & 37 & 37 & 37 & $37(37)$ \\
\hline
\end{tabular}




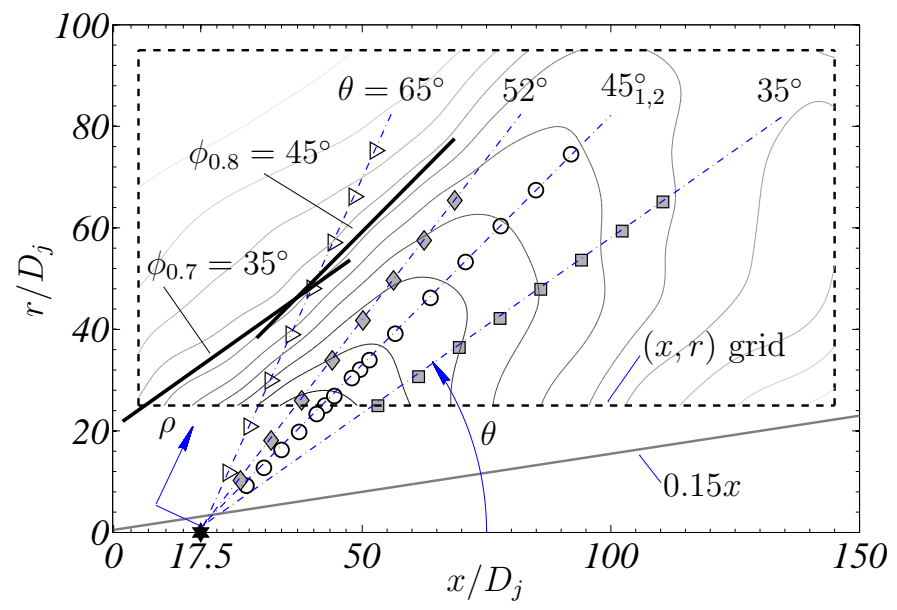

Figure 2.5: Location of $1 / 8$ inch microphone rays and coordinate system. Contour of OASPL $(\mathrm{dB}$, ref: $20 \mu \mathrm{Pa})$ from $1 / 4$ inch $(x, r)$ grid measurements of Baars \& Tinney (2014).

Nyquist frequency with the built-in anti-aliasing filters on the NI PXI-4472 card. The placement of these microphones involved both a 2-D grid of points in the $(x, r)$ plane as well as a line array along the peak emission path, which was found to emanate from a region centered around $x / D_{j}=17.5$. Spectral based metrics are constructed using $2^{13}$ samples per block, thus resulting in a frequency resolution of $\delta f=12.5 \mathrm{~Hz}$.

As for the current set of measurements, eight $1 / 8$ inch pressure-field microphones (G.R.A.S. Type 46DD capsules with G.R.A.S. Type 26CB preamplifiers) were placed at grazing incidence, as shown in Figure 2.1. These microphones are capable of resolving signals over a broader range of frequencies (from $6.5 \mathrm{~Hz}$ up to $140 \mathrm{kHz}$ with $\pm 2 \mathrm{~dB}$ error) with a dynamic range from 46 dB to $179 \mathrm{~dB}$ (ref:20 $\mu \mathrm{Pa}$ ). IEPE power was supplied by a Dytran Instruments Inc. 4121 power supply, which were then low pass filtered using a Khron-Hite 
corp filter (model FLX-3007). These low pass filters employ a $4^{\text {th }}$ order Butterworth filter and were custom designed to have a cut off frequency of 140 $\mathrm{kHz}$. Microphone signals were digitized simultaneously at $500 \mathrm{kS} / \mathrm{s}$ per channel using two NI PXI-6122 boards (four single ended channels per board with 16 bit resolution over $\pm 5 \mathrm{v}$ ) for 13.1 seconds uninterrupted. This largely oversampled rate ensures better resolution in the rise time of the shock structures, which is a crucial point in this study. One-sided power spectral densities were then generated from 400 blocks of data, each comprising $2^{14}$ samples per block and a frequency resolution of $\delta f=30.52 \mathrm{~Hz}$.

The 1/8 inch microphone measurements were conducted using four different rays centered on $x / D_{j}=17.5$, as is shown in Figure 2.5. For supersonic jets, the region in the flow between the collapse of the potential core and the supersonic core length are known to be the location where turbulent mixing is not only most intense, but also occurs at supersonic velocities [25,33]. Identifying the correct propagation path is not necessarily trivial as the jet does not behave like a single compact source. This is especially problematic close to the hydrodynamic region where pressure waves comprise a superposition of both acoustic and evanescent signatures [37]. Choosing the wrong path artificially triggers the appearance of nonlinear distortion in the signal that one might interpret as a sign of wave steepening; this problem has been addressed in past studies $[2,20]$. Here we attempted to place the microphones as close to the jet as possible without them being endangered, and without contaminating the signals with evanescent signatures. The jet shear layer growth coefficient was approximated to be 0.1 based on the measurements of Tinney et al. (2008) [38]. A more conservative estimate of 0.15 was employed as is shown in Figure 2.5. Table 2.2 identifies the location of the $1 / 8$ inch microphone rays and is listed 
Table 2.2: Locations of $1 / 8$ inch microphones.

\begin{tabular}{lcc}
\hline \hline$\theta$ & offset from source & $\Delta \rho$ \\
\hline $65^{\circ}$ & $13.0 D_{j}$ & $10 D_{j}$ \\
$52^{\circ}$ & $13.0 D_{j}$ & $10 D_{j}$ \\
$45_{1}^{\circ}$ & $13.0 D_{j}$ & $5 D_{j}$ \\
$45_{2}^{\circ}$ & $35.5 D_{j}$ & $10 D_{j}$ \\
$35^{\circ}$ & $43.5 D_{j}$ & $10 D_{j}$ \\
\hline \hline
\end{tabular}

in terms of the ray angle (measured relative to the jet centerline), the starting distance of each ray relative to the post potential core region, as well as the separation distance between subsequent microphones on each ray. 


\section{Chapter 3}

\section{Experimental results}

\subsection{Preliminary corrections and spectral observations}

Before we conduct a thorough analysis of the acoustic field, we have to perform some corrections of various nature to the dataset (this is the postprocessing step presented in diagram 2.4). First, a correction based on the manufacturer supplied data was performed to correct for the different frequency sensitivity of each microphone. As is shown in Figure 3.1b, the effect of this frequency correction on the spectral shape is subtle, but was used nonetheless. Then, in an effort to remove any possible contaminations produced by low frequency reflections in the chamber, a second order high-pass filter was applied to the data with a cut-off frequency of $300 \mathrm{~Hz}$; this correction is illustrated in Figure 3.1a. Additionally, comparative studies were performed with and without the microphone's protective caps, as well as without the lowpass antialiasing filter in order to gain an understanding of the influence these tools have on the acoustic field. The results are shown in Figure 3.2. It can be observed that the grid caps are having a negligible influence on the spectra, while the $140 \mathrm{kHz} 4^{\text {th }}$ order Butterworth filter is logically slightly starting to damp the spectra at the very high frequencies. We indeed measure a $3 \mathrm{~dB}$ difference at the cutoff frequency of the filter $(140 \mathrm{kHz})$. All the corrections

presented in this paragraph are found to only have a subtle influence on all the indicators which will be presented in the next chapter, but were used nonethe- 
less. Both the protective caps and high-pass/low-pass filters were applied for the remainder of the studies.

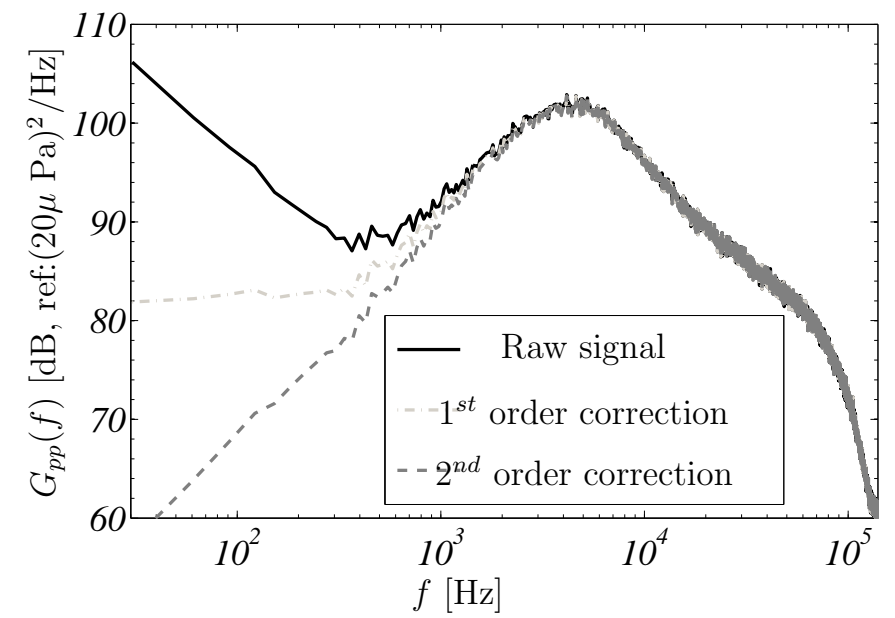

(a)

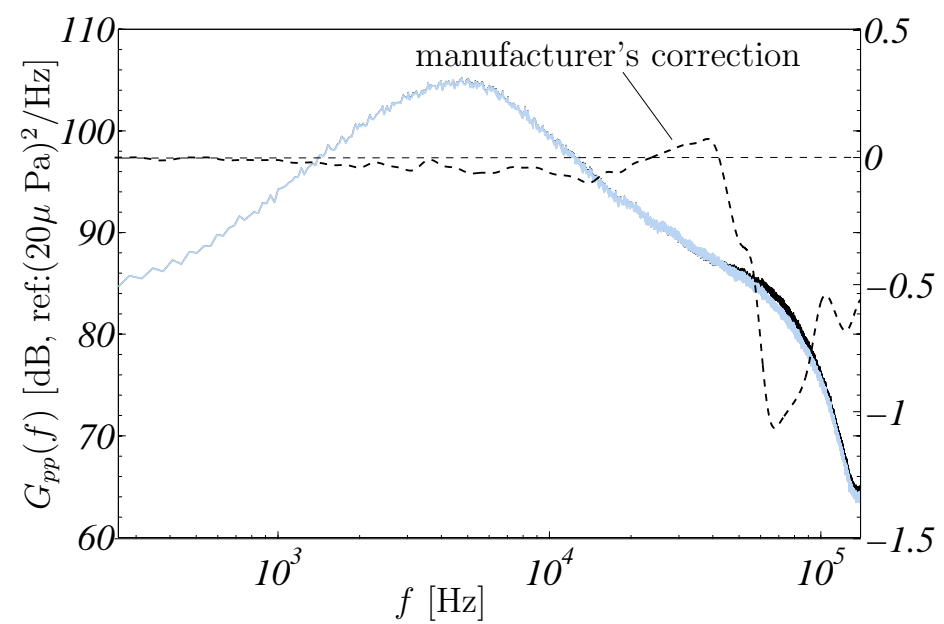

(b)

Figure 3.1: (a) One-sided power spectral density (PSD) with and without the $1^{\text {st }} / 2^{\text {nd }}$ order high-pass filter correction. (b) PSD of a sample microphone signal at $45^{\circ}$ with and without corrections using manufacturer supplied data.

An illustration of the final spectra is shown in Figure 3.3, with compari- 


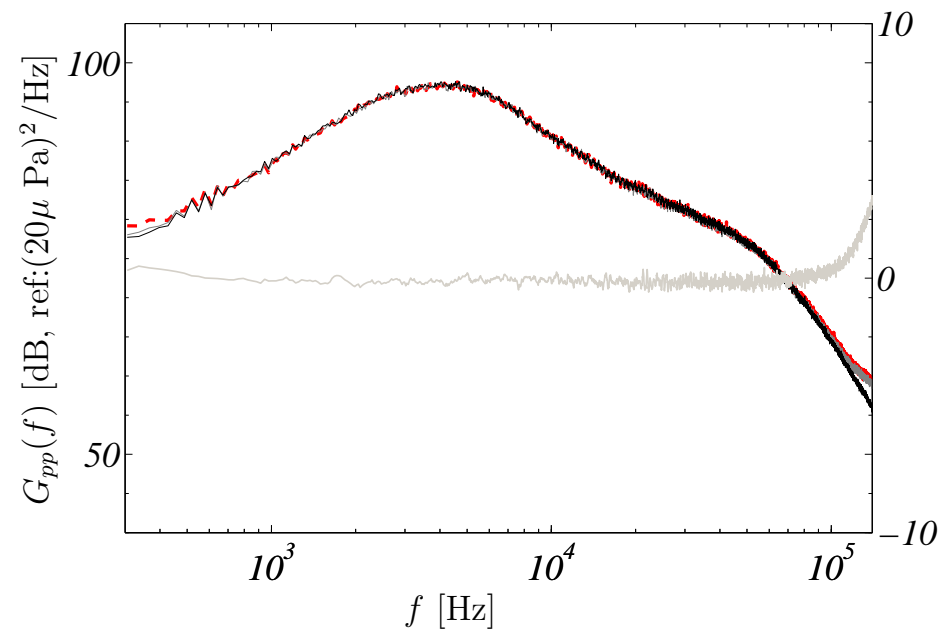

Figure 3.2: Spectral comparison with $4^{\text {th }}$ order Butterworth and grid caps off/off (red), off/on (dark gray) and on/on (black) for a microphone in the middle of the array $\left(\mathrm{x}=75 D_{j}\right)$; the light gray line shows the difference the filter causes to the spectrum.

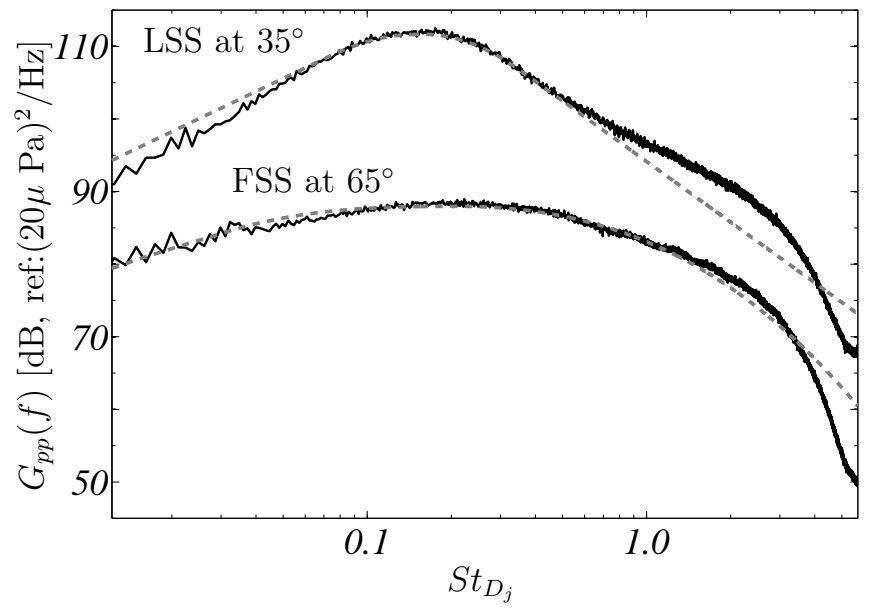

Figure 3.3: PSD at $\theta=35^{\circ}$ and $65^{\circ}$ being compared to the LSS and FSS of Tam et al. (1996).

son to the FSS and LSS spectra of Tam et al. (1996) [35]. The two microphones are selected from the $35^{\circ}$ and $65^{\circ}$ rays and are located at $85.3 D_{j}$ and $83.0 D_{j}$ 
from the jet source. The spectra corresponding to the $65^{\circ}$ observer are located outside of the Mach cone, and so they are characterized by a broader profile and a smooth roll-off, whereas at $35^{\circ}$ there is a noticeable characteristic peak with relatively linear decay. The good agreement between the experimental data and the similarity spectra is encouraging and demonstrates the universality of the spectra produced by high Mach number jet flows.

However, the trends used to create the LSS and FSS shapes are based on measurements where the jet exit plane was chosen as the propagation path. This was done using 1,900 spectra that covered a range of supersonic jet conditions where variations in Mach number and temperature ratio are concerned. And so, variations in the source region are expected, which were not corrected for by Tam et al. (1996) [35] when determining the true propagation path. Thus, discrepancies between the LSS and FSS trends, relative to the current data set could be attributed to a blending of far-field spectral shapes (due to changes in the emission path) that were used to generate the LSS and FSS shapes.

It is also relevant to observe the evolution of the spectra throughout the array. Figure 3.4a presents the spectra at all positions for two different testing sessions with identical experimental conditions, illustrating the repeatability of the experiment. Figure 3.4b shows the evolution of the acoustic spectrum. The shift in frequency that appears will be discussed in the next chapter when investigating for signs of cumulative nonlinear distortion.

\subsection{Sound pressure decay in the jet far-field}

Figures 3.5a and 3.5b present the decay and inverse decay, respectively, measured using the $1 / 8$ inch microphones along all four rays. The agreement 


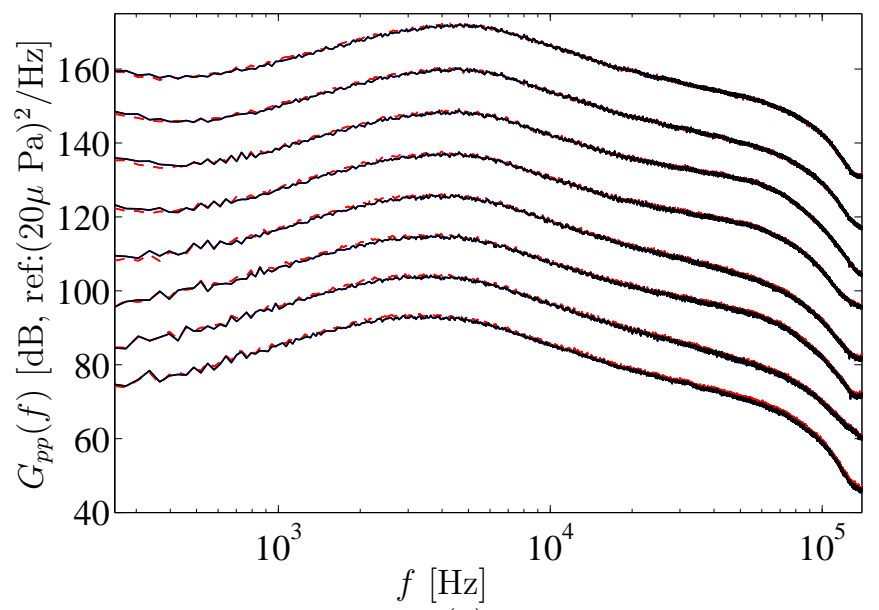

(a)

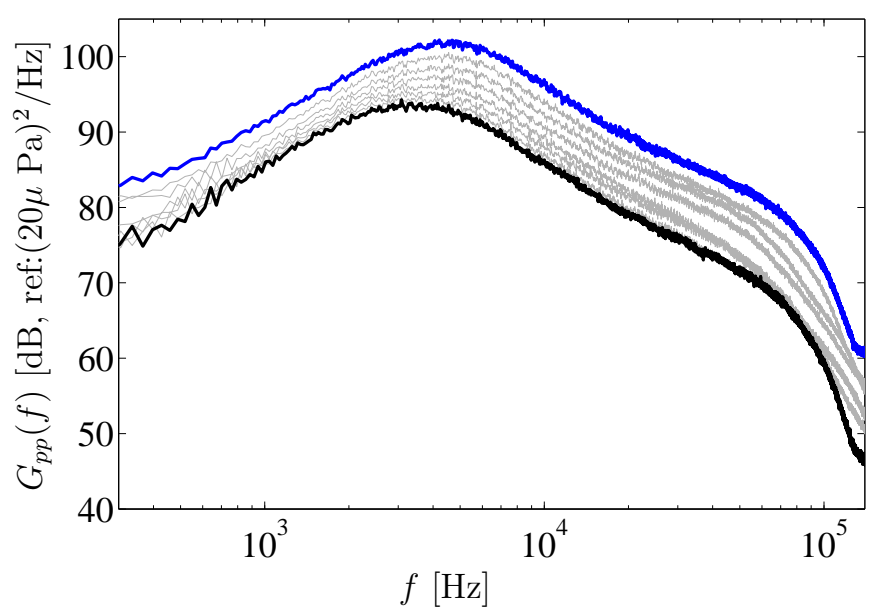

(b)

Figure 3.4: (a) PSD for two different testing sessions (black/red) with identical experimental conditions at all microphone positions along an array (spectra shifted by $10 \mathrm{~dB}$ ). (b) Evolution of the PSD along the second $45^{\circ}$ array (blue for the first microphone, black for the last one).

between the $45^{\circ}$ and $52^{\circ}$ rays is quite remarkable and confirms how linear decay holds well over a range of angles centered on the Mach wave angle. 
Thus one should expect some flexibility in both the convective speed of the relevant sound producing events, as well as the acoustic region where the propagation resembles that in a waveguide so to speak; spherical decay in the acoustic pressure is not restricted to a single angle. Albeit, the $45^{\circ}$ ray is the only arrangement where a higher spatial resolution permits a verification that this trend holds closer to the jet. Not so surprisingly is the fact that the rate of decay at steeper $\left(65^{\circ}\right)$ and shallower $\left(35^{\circ}\right)$ angles is much greater, the $65^{\circ}$ array being much more extreme. Glancing at the OASPL contours in Figure 2.5 justifies why this is expected.

A further observation is made with the decay trends presented in Figure 3.5 and in Figure 3.9 where the results are compared with those of Baars \& Tinney (2014) [4] (we will discuss the wave packet model in Section 3.3). Foremost, the remarkable agreement between the measurements acquired with the $1 / 4$ inch and 1/8 inch microphones demonstrates how transducer size and atmospheric absorption have little effect on the pressure variance (expressed here as OASPL). And so, the topography of the OASPL in Figure 2.5 using 1/4 inch microphones is considered an accurate mapping of the pressure intensity in the far-field of this Mach 3 jet flow. Likewise, because the sound pressure is shown to decay spherically after approximately 35 diameters, any prediction of the far-field pressure using measurements outside of this location, and assuming spherical decay, should provide an accurate mapping of the OASPL in the far-field, so long as the correct propagation path is employed. It is important that we emphasize how the location in which the acoustic pressure abides by spherical decay is based on the operating conditions of the nozzle and is expected to scale with acoustic Mach number. Most often the propagation path is chosen to emanate from the jet exit plane. Baars \& Tinney (2014) [4] 


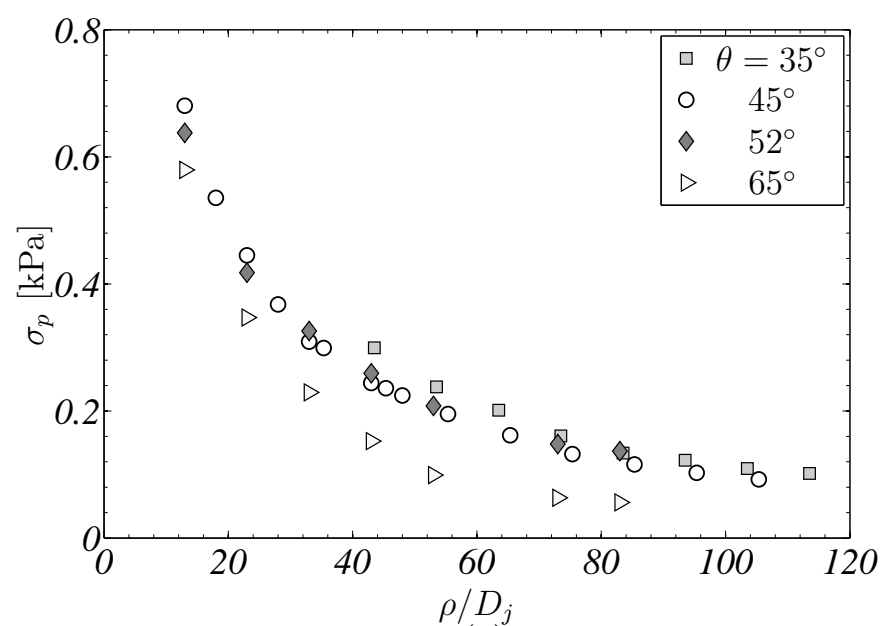

(a)

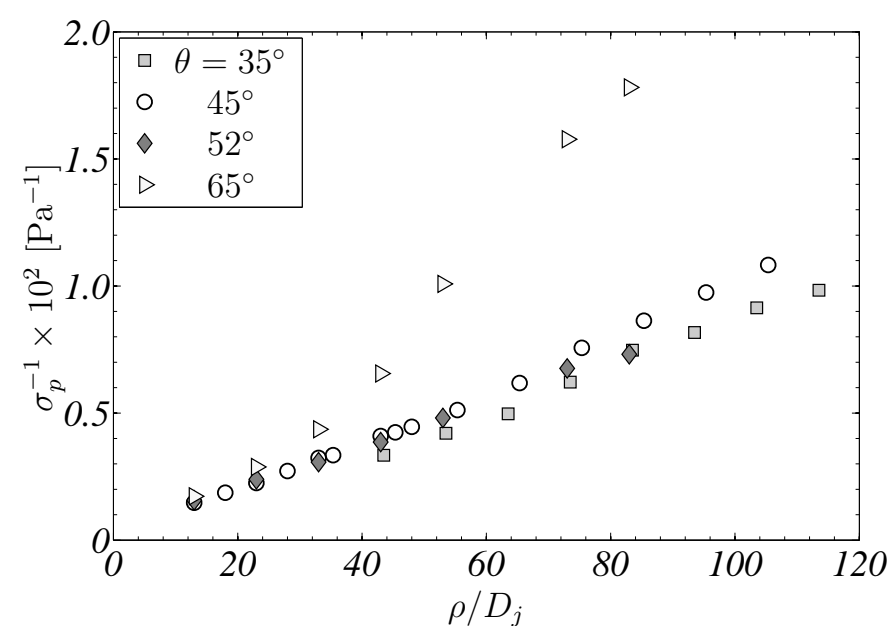

(b)

Figure 3.5: (a) $\sigma_{p}$ decay and (b) $\sigma_{p}^{-1} \times 10^{2}$ from $1 / 8$ inch microphones.

shows how various features in the far-field of supersonic jets collapse exceptionally well when the prominent source region, located after the collapse of the potential core, is chosen. A demonstration of this is shown in Figure 3.6 where the OASPL from the entire $(x, r)$ grid measurements of Baars \& Tinney (2014) [4] (120 measurement points in total) are extrapolated to an arc 


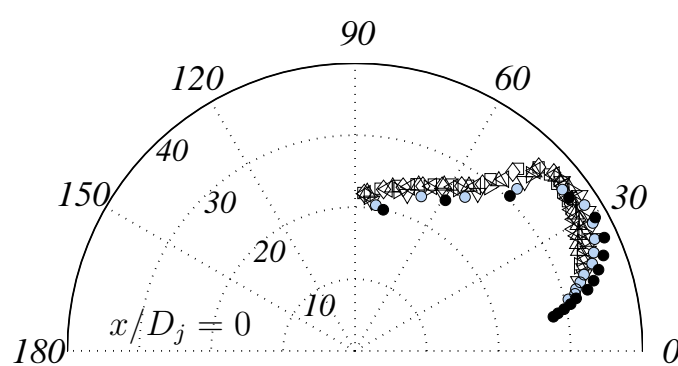

(a)

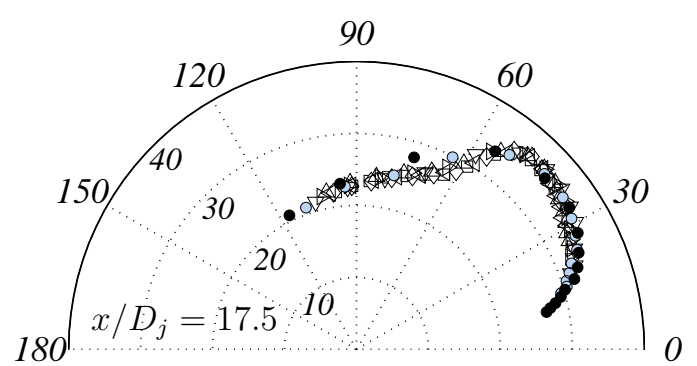

(b)

Figure 3.6: OASPL (dB, ref: $20 \mu \mathrm{Pa})$ from $(x, r)$ plane grid measurements of Baars \& Tinney (2014) projected to an arc array at $\rho / D_{j}=95$ using spherical decay. Assuming source region at (a) $x / D_{j}=0$ and (b) $x / D_{j}=17.5$. The values shown are less $100 \mathrm{~dB}$.

array situated at $\rho / D_{j}=95$ using spherical decay. In Figure 3.6a the source is assumed to reside at the nozzle exit plane at $x / D_{j}=0$, while in Figure 3.6b the source is moved downstream to $x / D_{j}=17.5$. The findings are remarkable and expose the kinds of gross errors that will occur when one chooses to propagate jet noise data along rays emanating from the jet exit plane; nearly identical observations have been made in the rocket noise community $[18,25]$. The only discrepancies in Figure $3.6 \mathrm{~b}$ are with the closer measurement points (identified as filled and open circles) that were located at $\rho / D_{j}<35$, and are anticipated since these waveforms initially undergo cylindrical decay (as shown in Figure 3.9), which was not corrected for in this simple exercise. Thus, both fine-scale similarity and large-scale similarity like regions in the far-field can be predicted from measurements closer to the jet itself, so long as the correct propagation path is employed. 


\subsection{Wave packet}

One of the underlying challenges in implementing the model developed by Baars (2013) [3], which predicts shock formation distances and the degree of cumulative nonlinear distortion in the acoustic waveform from high speed jets, is that it requires one to know the size and amplitude of the source. This is a difficult proposition, even with the advent of more sophisticated numerical simulations at our disposal; consider the different source definitions floating about the literature $[13,23,24]$. The experimental handicap is pretty straightforward: simply placing a pressure sensing element closer to a jet flow manifests a flow-biased description of the source properties, given the blending of both acoustic and hydrodynamic waves within the jet's hydrodynamic periphery. Two established methods, one described by Tinney \& Jordan (2008) [37], the other by Grissi \& Camussi (2012) [14], offer some relief in separating these two components of the pressure field, but are limited to flows comprising soundproducing events with convective speeds that are, on average, subsonic. And so, an adequate means by which the source mechanisms, responsible for generating Mach waves, can be isolated and studied remains to be found.

A resurgence in recent years with the use of wave packets (Morris (2009) [29], Papamoschou (2011) [31], Kuo et al. (2012) [21] and Jordan \& Colonius (2013) [19]) may offer some relief, and at a reduced cost. Modeling the evolution of supersonically convecting large-scale instabilities by wave packets provides a theoretical framework for studying the noise radiated by supersonic jets. In Figure 3.7 (taken from Appendix A of Baars [3]), the standard deviation $\left(\sigma_{p}\right)$ of the radiating component of the pressure signal along the Mach wave radiation angle, and emanating from the source region, is shown to encompass three distinct zones: one that abides by cylindrical decay 
$\left(\sigma_{p} \propto \rho^{-0.5}\right)$, another that abides by spherical decay $\left(\sigma_{p} \propto \rho^{-1.0}\right)$ and a third intermediate zone that transitions the two. Certainly the location where this transition occurs is quite important in determining source amplitude. Baars (2013) [3] shows how the answers to this can be expressed as a ratio of the wave packet model scale $(L)$ relative to the jet diameter, such that $A=L / D_{j}$; the effect of $A$ on the three decay regions is shown in Figure 3.7 for a source located on the lip line of the nozzle. Thus, to determine the proper values associated with both the wave packet scale and its location $\left(r_{w p}\right)$, a sensitivity study was conducted. For this, the spatial limits of the Gaussian envelop for all cases was confined to three standard of deviations while the wave packet amplitude was adjusted using pressure levels measured halfway along the array. Wave packet parameters $r_{w p}$ and $A$ that were finally chosen were based on a least error sum between all available microphones and the wave packet. The error function expression for this is defined as follows:

$$
W_{\text {error }}=\sum_{i=1}^{N} \frac{\left[\left(w_{i}-m_{i}\right)(N-i)\right]^{2}}{m_{i}^{2}}
$$

where $w_{i}$ corresponds to the wave packet pressure value located at $m_{i}$ and $i$ increases with increasing distance from the source. Furthermore, measurements closer to the jet were weighted more heavily (using $N-i$ ) than measurements further away, given the larger changes in pressure amplitude that occur closer to the source region. The results of this sensitivity study are shown in Figure 3.8 where the location of the source is expressed by its position along the propagation path $\left(\rho_{0}\right)$. Additional lines have been added which identify the final wave packet size $(A=8.75)$ that was selected for the remainder of the analysis, as well as the location of the jet shear layer. A value of $\rho_{0}=(\sqrt{2} / 2) D_{j}$ was also chosen, placing the wavepacket along the nozzle lip line. Thus, the 
large-scale instability waves are assumed to initiate their development at the exit plane of the nozzle and grow along the nozzle lip line to their peak level at $x / D_{j}=17.5$ before decaying to negligible levels far downstream. Under these assumptions, $2 L=17.5$. In Figure 3.9, the measured pressure decay (using both the $1 / 4$ inch and 1/8 inch microphones placed along the $45^{\circ}$ path) compares favorably with the wave packet model. Deviations from the cylindrical and spherical decay laws are found to be within $2 \%$ of the wave packet model at $\rho / D_{j}=12.3$ and 33.4 , respectively. The findings are a testament to both the scale of the wave packet chosen, as well as the propagation path along which the microphones are placed. 


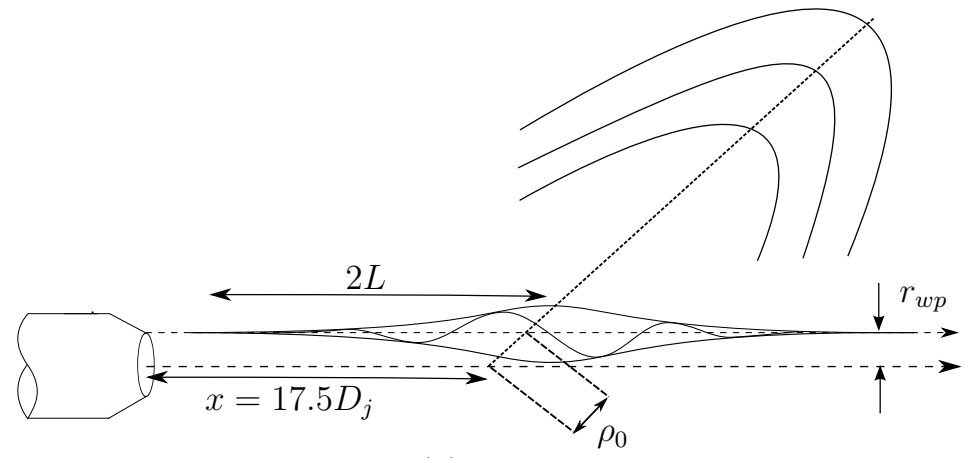

(a)

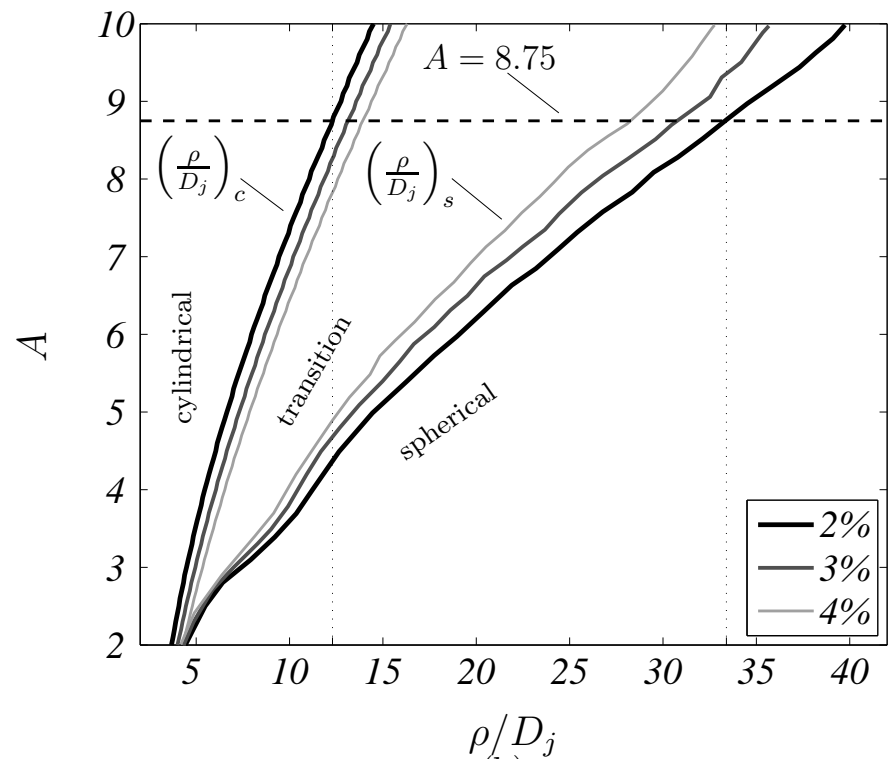

(b)

Figure 3.7: (a) Schematic of the wave packet scale versus jet scale. (b) Regions where the wave packet model obeys cylindrical and spherical spreading along the peak noise path. Contour lines define error thresholds of $2 \%, 3 \%$ and $4 \%$. 


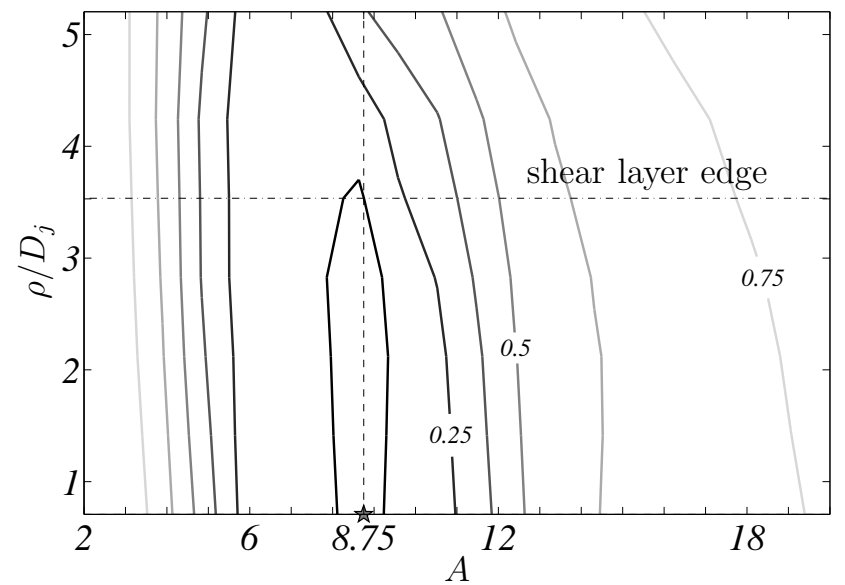

Figure 3.8: Integrated errors between the wave packet model and laboratory measurements for various wave packet length scales and source locations along the propagation path. Contour levels have been normalized so that they range between 0 and 1 with increments of 0.125 .

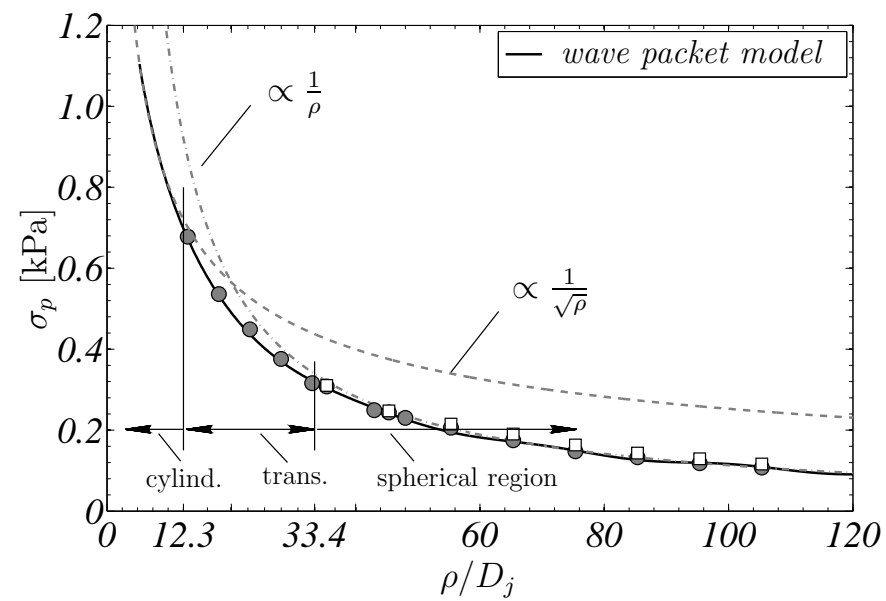

Figure 3.9: Spreading trends along the peak noise emission path obtained from the wave packet model using $A=8.75$. Both experimental datasets are shown with $\square$ corresponding to the $1 / 4$ inch microphones. 


\section{Chapter 4}

\section{Analysis of acoustic waveforms along the peak emission path}

Baars \& Tinney (2014) [4] demonstrated how the statistical properties of the acoustic waveforms are relatively unchanged along rays emanating from the prominent source regions of supersonic jets. Furthermore, only weak waveform distortions were measured using $1 / 4$ inch microphones placed in the mid to far-field regions of the jet and under higher levels of humidity. And so, the motivation of this part of our study is twofold. The first is to demonstrate the effect of sensor size and atmospheric absorption on capturing these distortions, while the second is to address the postulations of Baars \& Tinney (2014) [4] through a thorough analysis of the data acquired with the higher fidelity instruments.

The statistical metrics that are employed comprise the quadrature spectral density, skewness of the pressure time derivative, the number of zero crossings per unit time $\left(N_{z c}\right)$ and the integral of the negative component of the quadrature spectral density. These metrics are used to determine the degree of nonlinearity in the acoustic waveform at a single point. When analyzed over several points along the propagation path, the changes to the waveform amount to nonlinearities accumulated from cumulative distortion. This is true so long as the measured waveforms are from a single source, or have limited interference from neighboring wavefronts produced by other nearby sources in 
the flow. The latter could give rise to artificial steepening through constructive interference, forming rogue wave shocks contaminating the statistics. Other metrics were considered and included the wave steepening factor and Kurtosis, though neither revealed anything significant and so they are not shown here. A listing of these metrics is provided as follows:

- Quadrature Spectral Density. The definition for this metric is well documented in the work of Morfey \& Howell (1981) [28] and Howell \& Morfey (1987) [17]. This quantity highlights the importance of non-linear distortion across the spectrum relative to the power spectral density; the normalized forms of this indicator is as follows,

$$
\begin{gathered}
Q=\frac{Q_{p^{2} p}}{\sigma_{p}^{3}} \\
\frac{Q}{S}=\frac{Q_{p^{2} p} / \sigma_{p}^{3}}{S_{p p} / \sigma_{p}^{2}}
\end{gathered}
$$

where $Q_{p^{2} p}=-\Im\left\langle 2 \hat{p}_{2}(f) \hat{p}^{*}(f)\right\rangle$, and $\hat{p}(f)$ and $\hat{p}_{2}(f)$ are the Fourier transforms of the signals $p(t)$ and $p^{2}(t)$, respectively. The denominator in Eq. (4.2) comprises the ensemble averaged power spectral density, which is simply $S_{p p}=\left\langle\hat{p}(f) \hat{p}^{*}(f)\right\rangle$. Howell \& Morfey (1987) [17] recognized early on the limitation in using these metrics at a single point and that the problem [of predicting distortion] remains unsolved without estimating $Q_{p^{2} p}$ as a function of distance. This is especially problematic with experimental data, where transducer errors inherently prevent the signal from being truly Gaussian; thus it is difficult to envision this term ever being zero in a laboratory environment. Figure 3.5a illustrates how the pressure standard deviation along the $45^{\circ}$ ray abides by realistic decay 
laws, and so an application of both Eqs. (4.1) and (4.2) to this data should provide meaningful results.

- Skewness of the pressure derivative. The amount of wave steepening, and thus the degree of nonlinearity in the pressure signal, can be quantified by computing the probability density function (PDF) and skewness factor of the pressure time derivative $\dot{p}(t)$. The PDF is denoted as $B(\dot{p})$ while its asymmetry is defined by the third central moment,

$$
\overline{\dot{p}^{3}} \equiv \int_{-\infty}^{\infty} \dot{p}^{3} B(\dot{p}) d \dot{p} .
$$

The dimensionless asymmetry is thus the well known skewness factor, and is defined as

$$
\gamma \equiv \frac{\overline{\dot{p}^{3}}}{\dot{\sigma}_{p}^{3}},
$$

where $\dot{\sigma}_{p}^{2}$ is the variance of $\dot{p}(t)$. Numerous applications using this metric to quantify nonlinearities in real waveforms can be found in the literature with the skewness of the pressure time derivative providing the most sensitivity [26].

- Kurtosis of the pressure derivative. The sharpness of waveforms can be quantified by computing the fourth central moment of the signal, which is known as the kurtosis. A value of 3 corresponds to a signal with a Gaussian distribution. Like what was done for the skewness, it is more suitable to apply the kurtosis metric to the time derivative of the pressure signal. The time derivative of a signal containing saw-tooth like waveforms (sharp edges, followed by long tails with a small slope) consists in sharp peaks with low negative constant values in between. A large 
kurtosis factor is an indicator of such isolated sharp peaks. It therefore serves as an indicator of shock formation and also as an indicator of shock coalescence since the kurtosis metric will rise as the distance between successive peaks increases.

It is defined as

$$
\kappa \equiv \frac{\overline{p^{4}}}{\dot{\sigma}_{p}^{4}}
$$

- Number of zero crossings per unit time. Since higher amplitude waves move faster along the propagation path and merge or coalesce with weaker waveforms, the number of zero crossings will decrease with increasing distance from the source. This does not necessarily mean that the waveform is steepening along the propagation path and so the link between $N_{z c}$ and cumulative nonlinear distortion effects warrants caution.

- Integral of negative $Q$. If cumulative distortions are occurring along the propagation path, then one would anticipate a flux of energy in and out of the mid frequencies. This can be quantified by integrating the negative part of the quadrature spectral density at each location on the ray as follows,

$$
Q_{n e g}(\rho)=\int_{Q<0} Q(f, \rho) d f .
$$

\subsection{Spectral indicators}

Profiles of $Q$ and $Q / S$ are shown in Figures 4.1a and 4.1b using Eqs. (4.1) and (4.2), respectively, along the four different rays (a 1\% bandwidth moving 
filter has been employed). As expected, both $Q$ and $Q / S$ profiles reflect a loss in energy at mid frequencies, with $Q / S$ demonstrating an increase in energy at high frequencies. Closer inspection reveals two distinct trends. The first comprises grey lines with arrows indicating the direction of increasing distance from the source and with noticeable changes in both energy and peak frequency. Increases in mid-frequency energy suggest waveform coalescence while increases in high frequency energy suggests waveform steepening. The other set, highlighted by solid dark lines, encompasses regions that reside beyond approximately $40 D_{j}$. These black lines collapse reasonably well and suggest that the acoustic waveforms are no longer coalescing or distorting along the propagation path; this agrees well with the measurements of Baars \& Tinney (2014) [4] using the $1 / 4$ inch microphones. Along the $65^{\circ}$ ray, the trends are difficult to decipher. Since this ray is outside the Mach cone, the lack of any discernible trend is expected. Albeit, the near perfect collapse of the OASPL shown in Figure 3.6b is a testament to the choice of propagation path, even for the sideline and steep angle observer. This is consistent with the measurements of Baars \& Tinney (2014) [4] where it has been shown that shock structures are concentrated mainly along the Mach wave angle.

Figure 4.2 presents both $Q$ and $Q / S$ spectra before and after the bandwidth moving filter is applied. It shows both the interest and the validity of using such filter for illustration purposes.

\subsection{Scalar indicators}

Figure 4.3 compares the number of zero crossings per second, the skewness and kurtosis of the pressure derivative and the integral of $Q_{n e g}$ along the $45^{\circ}$ path. The data acquired with the $1 / 8$ inch microphones are analyzed using 


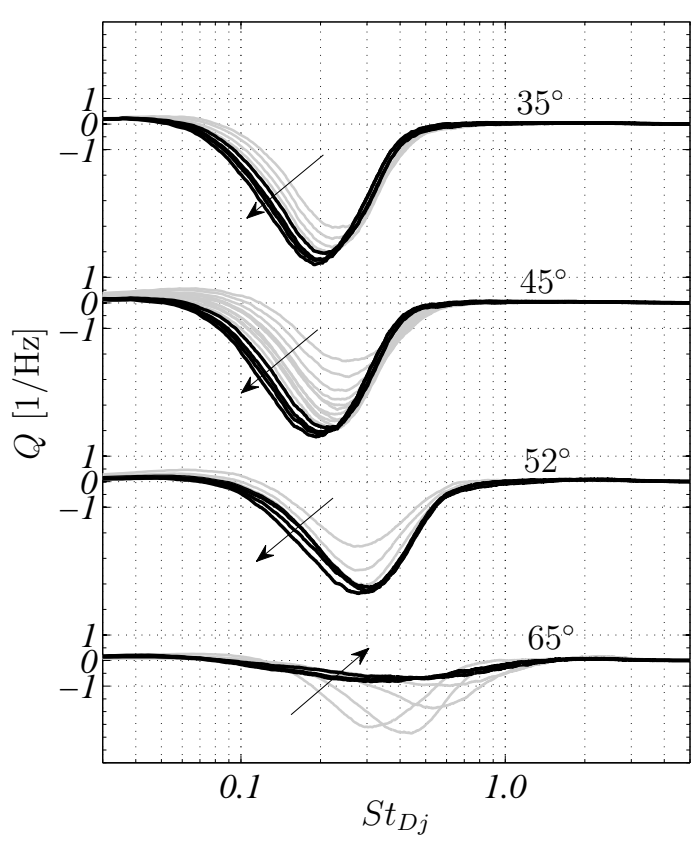

(a)

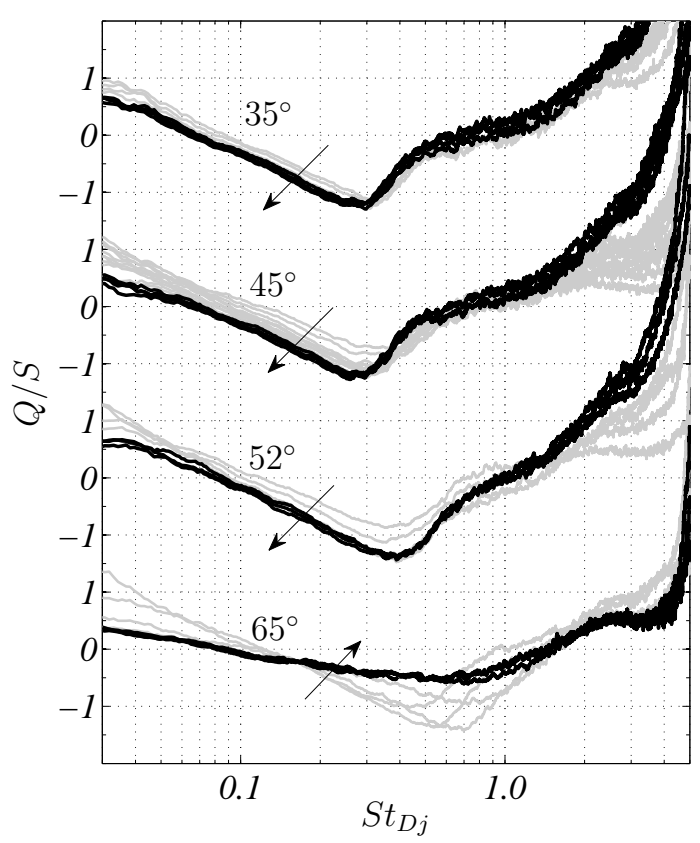

(b)

Figure 4.1: (a) $Q \times 10^{-5}$ and (b) $Q / S$, Morfey-Howell indicators (1\% bandwidth moving filter) for all microphones (gray) and for the last 4 microphones (black).

both the original sample rate of $500 \mathrm{kHz}$ as well as a reduced rate of $100 \mathrm{kHz}$, which coincides with the sample rate used by the $1 / 4$ inch microphones. The findings from the latter are used to compare the $1 / 8$ inch microphone measurements with the data acquired using the $1 / 4$ inch microphones (except for the kurtosis) in an effort to determine the effect of transducer size and atmospheric properties (humidity and temperature) on these higher order metrics. For each metric, an arbitrary approximation of the trend corresponding to the $500 \mathrm{kHz}$ sampling of the $1 / 8$ inch microphone is shown, along with its spatial derivative. These derivatives reveal three distinct zones. The first manifests a 


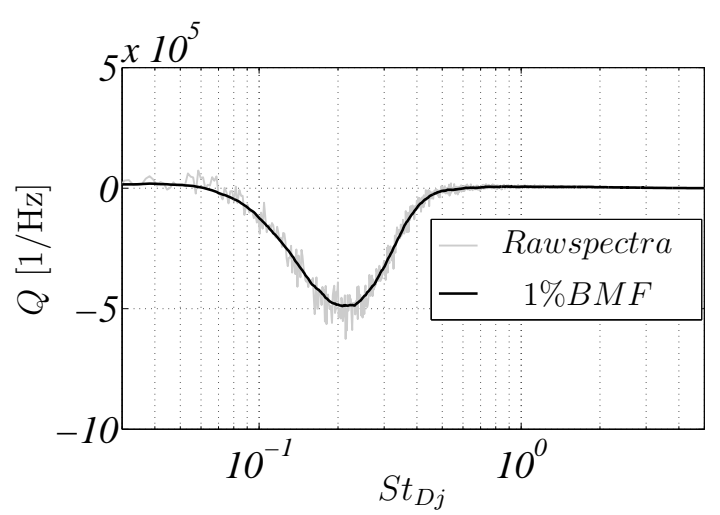

(a)

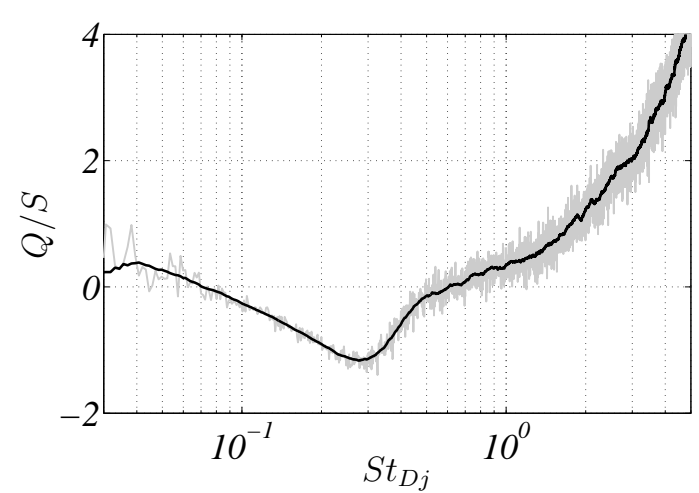

(b)

Figure 4.2: (a) $Q$ and (b) $Q / S$ with and without the $1 \%$ bandwidth moving filter $(\mathrm{BMF})$.

fairly constant spatial rate of change while the second reveals changes in amplitude along the propagation path. The third zone identifies a region where the metric is saturated and is no longer changing.

Where the number of zero crossings the skewness and the kurtosis of the pressure time derivative are concerned, the effect of sampling rate is clearly manifest. However the effects of temperature and humidity on the number of zero crossing and the integral of $Q_{n e g}$ appear not to be affected. While corrections for atmospheric absorption are well established, they cannot be corrected in the raw time signal. And so the effects of atmospheric absorption can only be ascertained from raw measurements of this kind.

As for the skewness of the pressure derivative $\gamma(\dot{p})$, several relevant features are seen in Figure 4.3b. First of all, three trends corresponding to each data set (same legend as in Figure 4.3a) are clearly present. The new data set sampled at $500 \mathrm{kHz}$ shows a quasi-linear growth of $\gamma(\dot{p})$ that suggests wave steepening; zero skewness is reflective of a normal distribution. By 80 
or so jet diameters $(\rho=2 \mathrm{~m})$ the steepening process appears to have reached its maximum at which point the trend remains either constant or begins to decay. For the resampled data (1/8 inch microphones resampled at $100 \mathrm{kHz}$ ), the change in $\gamma(\dot{p})$ follows the originally sampled data consistently up until roughly 0.7 meters from the source region, after which changes in the skewness begin to reflect the trends produced by the $1 / 4$ inch microphone study. This agreement demonstrates how distortion mechanisms affect the low frequency bands in the waveform earlier on as the lower sampled data reflects that of the higher sampled data. Differences between the resampled 1/8 inch microphone data and the $1 / 4$ inch microphone measurements are attributed to elevated levels of humidity during the $1 / 4$ inch study. A visual extrapolation of the trends toward the origin $(\rho \rightarrow 0)$ suggests that the waveforms produced in the source region of the jet are already skewed, thus confirming the simulations of Nichols et al.(2013) [30]. Very similar results are found regarding the evolution of $\kappa(\dot{p})$ along the propagation path, though the roll-off region appears at the end of the array, in the last $20 D_{j}$.

Figure 4.3d quantifies the net flux of energy transfer across the quadrature spectral density shown in Figure 4.1a. By restricting the limits of integration to only the negative part of the spectra, which is shown to be concentrated on the mid frequency range, any noise inherent to the borders of the spectrum are avoided. This registers the flux of mid-range spectral energy that is lost to waveform coalescence and is therefore a relevant indicator. Once again, a trend very similar to $\gamma(\dot{p})$ appears with a noticeably linear growth at the start of the array. Further away the evolution starts to decrease and eventually converges as the spatial rate of change becomes negligible.

The evolution of the spatial rate-of-change along the array for these 
four indicators are shown alongside one another in Figure 4.3e with each one being normalized by its maximum amplitude in order to compare trends. Overall, these indicators comprise a similar shape, which would suggest that both waveform steepening and coalescence are occurring within 2.5 meters or so from the source region of this laboratory scale jet.

\subsection{Shock formation distance}

In an effort to link the observed phenomena to the theoretical estimates for shock formation distance, two such formulations are considered, one for spherically propagating waves and the other for cylindrical waves. Given the changes in pressure amplitude registered by our measurements and confirmed by wave packet modeling, the real solution will be a blended estimate of the two. Following Hamilton \& Blackstock (2008) [15], the shock formation distance for an initially broadband Gaussian waveform that propagates cylindrically and spherically from its source in a loss-less fluid can be estimated from, respectively,

$$
\bar{r}_{c}=r_{0}\left(1+\bar{x} / 2 r_{0}\right)^{2}
$$

and

$$
\bar{r}_{s}=r_{0} \exp \left(\bar{x} / r_{0}\right)
$$

where $\bar{x}$ is the plane wave shock formation distance defined by Gurbatov \& Rudenko (p.383 of Hamilton \& Blackstock [15]) as,

$$
\bar{x}=\frac{\rho_{\infty} a_{\infty}^{3}}{\beta\left(2 \pi f_{0}\right) \sigma_{p, 0}},
$$

and $r_{0}$ is the source radius. The coefficient of nonlinearity is given by $\beta=$ $(\gamma+1) / 2=1.2$, with $\gamma$ being the heat capacity ratio for a perfect diatomic gas. 
while the density of air is determined from Table 2.1 and ideal gas properties to be $\rho_{\infty}=1.14 \mathrm{~kg} / \mathrm{m}^{3} . \sigma_{p, 0}$ is the standard deviation of the pressure time signal at the source. Figure 3.3 reveals a characteristic frequency valued at $f_{0}=3.6$ $\mathrm{kHz}$ for the $45^{\circ}$ ray, which is the same used by Baars (2013) [3]. Both the size and strength of the source are not easily determined, given the experimental and numerical handicaps previously discussed. Likewise, expressions for shock formation distance require that $k r \gg 1$, that is, the source radius must exceed the maximum characteristic wavelength of the radiated sound. Three likely locations for the source surface are considered in this exercise, though an infinite number of solutions can be obtained. The first borders the periphery of the jet shear layer at $\rho=3.5 D_{j}$ by assuming a shear layer growth coefficient of 0.1 [38]. The second is selected to replicate the location used by Baars (2013) [3] so that $r_{0}$ is located at $\rho=2.5 D_{j}$. The third location is assumed to be located on the nozzle lip-line at $\rho=(\sqrt{2} / 2) D_{j}$, where the peak noise source has been shown to reside [11]. Each of these locations is within the cylindrical decay region of the pressure amplitude, and so the source amplitudes (for these three points) are extrapolated using the wave packet solution for $L=8.75$ and assuming $p \propto 1 / \sqrt{\rho}$. Shock formation distances are then estimated using Eq. (4.7) and (4.8) for cylindrical and spherical decay along the entire propagation path, respectively. Thus, for a starting waveform that abides by Gaussian statistics and is allowed to propagate in the absence of viscous absorption effects, its shock formation distance will be bounded by $\bar{r}_{c}$ and $\bar{r}_{s}$.

Findings from this analysis are shown in Table 4.1 alongside the original estimates from Baars (2013) [3] who estimated the source amplitude to be a factor of three larger than what is measured here; Baars (2013) [3] assumed the source amplitude abides by spherical propagation along its entire path, 
Table 4.1: Shock formation distances and effective Gol'dberg numbers.

\begin{tabular}{l|ccc|c}
\hline \hline$r_{0}, D_{j}$ & 0.7 & 2.5 & 3.5 & 2.5 (Baars 2013) \\
\hline$k r_{0}$ & 1.2 & 4.1 & 5.8 & 4.2 \\
$\sigma_{p, 0}, \mathrm{~Pa}$ & 2900 & 1546 & 1300 & 4948 \\
\hline $\bar{x}, \mathrm{~m}$ & 0.62 & 1.17 & 1.39 & 0.36 \\
$\bar{r}_{c}, \mathrm{~m}$ & 6.04 & 6.60 & 6.85 & 0.92 \\
$\bar{r}_{s}, \mathrm{~m}$ & $10^{13}$ & $10^{7}$ & $10^{5}$ & 17.2 \\
\hline$\Gamma$ & 618 & 329 & 277 & 1171 \\
$\Lambda_{c}$ & 22.9 & 21.3 & 21.1 & 217 \\
$\Lambda_{s}$ & $10^{-21}$ & $10^{-10}$ & $10^{-8}$ & 0.18 \\
\hline \hline
\end{tabular}

which is reasonable, considering the information that was available at the time. Nonetheless, the recipe outlined by Baars (2013) [3] is employed here and provides reasonable estimates for $\bar{r}_{c}$ and $\bar{r}_{s}$. The findings suggest that, aside from both the effects imposed by having presteepened waveforms at the source as well as viscous relaxation along the propagation path, it is unlikely that shocks will form within the confines of this laboratory facility.

\subsection{Gol'dberg number}

The next step is to calculate the Gol'dberg number $\Gamma$, a measure of the strength of nonlinear distortion relative to that of dissipation. When $\Gamma<1$, attenuation dominates and the formation of shocks is suppressed. When $\Gamma \gg 1$ nonlinear effects will be distinctly present. The expression for plane waves is well established and is defined as,

$$
\Gamma=\frac{\ell_{\alpha}}{\bar{x}}
$$

where the absorption length scale $\left(\ell_{\alpha}\right)$ is simply the inverse of the absorption coefficient $(\alpha)$ which is evaluated in Table 4.1 using an expression from Bass 
et al. (1996) [6]. We evaluate $\alpha$ using the characteristic frequency $f_{0}=3.6$ $\mathrm{kHz}$ and for a relative humidity determined by the average value recorded during the $45_{1,2}^{\circ}$ tests $(\% \mathrm{RH}=45)$. The resultant absorption for the $1 / 8$ inch microphone study is found to be $\alpha=0.0026 \mathrm{~Np} / \mathrm{m}$, which yielded $\ell_{\alpha}=385$ $\mathrm{m}\left(\ell_{\alpha}=417 \mathrm{~m}\right.$ for the $1 / 4$ inch microphone study conducted by Baars et al. (2012) [2] based on 70\% relative humidity and an atmospheric temperature of $290 \mathrm{~K})$. Surprisingly, the differences in absorption between the current measurements and the $1 / 4$ inch study is small; changes in temperature are balanced by changes in humidity for this particular frequency. As was done for the shock formation distance, an expression for the Gol'dberg number for cylindrically and spherically propagating waves is needed. Hamilton (2013) [16] recently showed effective Gol'dberg number for diverging waves can be expressed as

$$
\Lambda_{c}=\frac{\Gamma}{1+\pi \bar{x} / 4 r_{0}}
$$

or

$$
\Lambda_{s}=\Gamma \exp \left(-\pi \bar{x} / 2 r_{0}\right)
$$

where Eq. (4.11) and (4.12) are the cylindrical and spherical forms of the expression. Estimates of $\Lambda_{c}$ and $\Lambda_{s}$ for the $1 / 8$ inch and $1 / 4$ inch studies are provided in Table 4.1. That is, estimates that assume purely cylindrical decay suggest cumulative nonlinear distortions will be present in the waveform, whereas estimates assuming pure spherical decay suggest that no such distortions will form.

\subsection{Direct observation}

A comparison of the instantaneous waveforms registered along the $45^{\circ}$ ray in the inner and outer zones are shown in Figures 4.4 and 4.5, respec- 
tively. These waveforms are aligned by cross-correlating the first microphone signal with subsequent signals on the array, where $\xi_{p p^{\prime}}(\tau)=\left\langle p(\rho, t) p\left(\rho^{\prime}, t\right)\right\rangle$. The corrected time delay is identified by the maximum correlation, as shown in Figure 4.6, which exposes two important features. That is, correlations of this magnitude demonstrate how directive the waveforms are along this path, while the decay in correlation level with increasing distance is evidence of contamination from other waveforms produced by other sources located elsewhere in the flow. And so, this raises additional concerns as to whether cumulative waveform distortions are forming beyond the hydrodynamic near-field regions of laboratory scale jets, aside from the statistical analysis provided in Section 4.1.

Turning our attention back to the raw pressure time series in Figures 4.4 and 4.5, several features are manifest, which complement the simulations of Nichols et al. (2013) [30] and Anderson \& Freund (2012) [1]. Albeit, without knowing the particle velocity simultaneously, the discussion and interpretation provided here warrants caution. In Figures 4.4 and 4.5, several additional lines have been drawn with labels corresponding to the following kinds of events:

- Wave Steepening (WS). This corresponds to instances when the wave registered at multiple locations on the ray appears to be the same wavefront and is steepening. This is identified by a single wave that evolves from a more relaxed wave shape to a sawtooth shape with increasing distance.

- Wave Passing (WP). These waves correspond to instances where the direction of propagation differs slightly from the $45^{\circ}$ path along which the microphones are placed. Because the width of the wavefront is finite, 
it either appears or disappears in the microphone signal at various points consecutively. It is assumed that the number of waves entering and exiting the propagation path is the same.

- Rogue Wave (RW). These will form when two smaller waveforms travel at small angles relative to one another and eventually merge to produce a much larger and steepened wave whose amplitude is approximately that of the original waves combined.

- Wave Coalescence (WC). This is similar to a rogue wave where two waves, propagating along the same path, merge and then coalesce.

The significance of having both passing waves and rogue waves occurring along the propagation path is that both will artificially increase $N_{z c}$, $\gamma(\dot{p}), \kappa(\dot{p})$ and $Q_{n e g}$ and mislead one into believing that cumulative distortions are occurring along the propagation path. For the sample time series shown in Figures 4.4 and 4.5, instances of passing waves is significant. Rogue waves only appear in these time series at further distances in Figure 4.5 where sawtooth-like structures are also significant. Two samples of rogue and passing waves are shown in Figure 4.7. We can observe in Figure 4.7a that the shock which is detected at the first microphone seems to actually be a constructive interference of two smaller waveforms which separate from each other while they propagate to the far-field. Figure $4.7 \mathrm{~b}$ shows the sudden appearance of a sharp shock from a low-amplitude signal : this shock hasn't been created by distortion along the microphone path, it is just passing through it. 


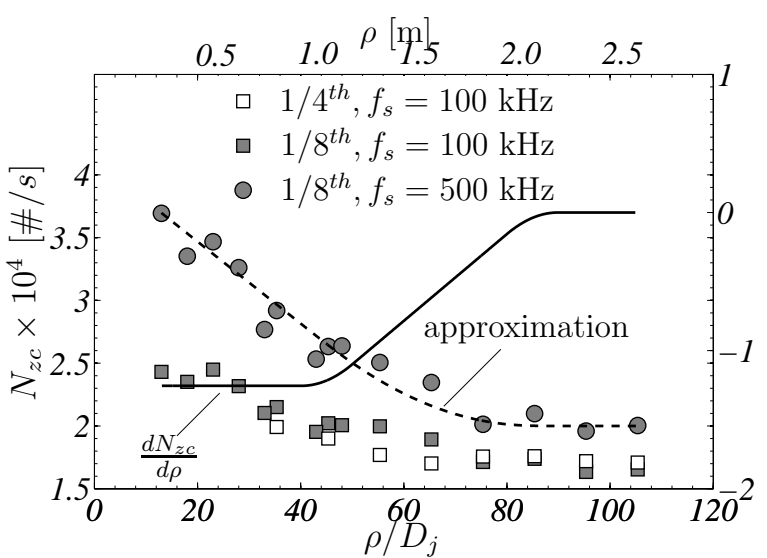

(a)

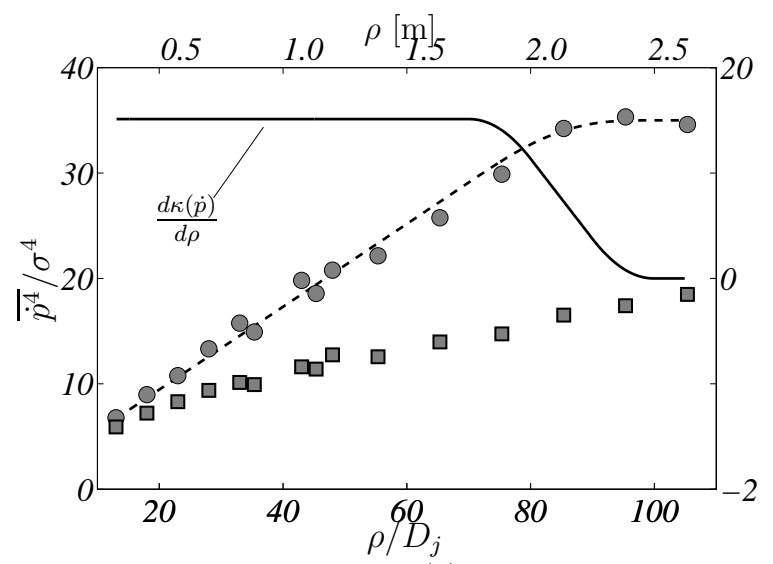

(c)

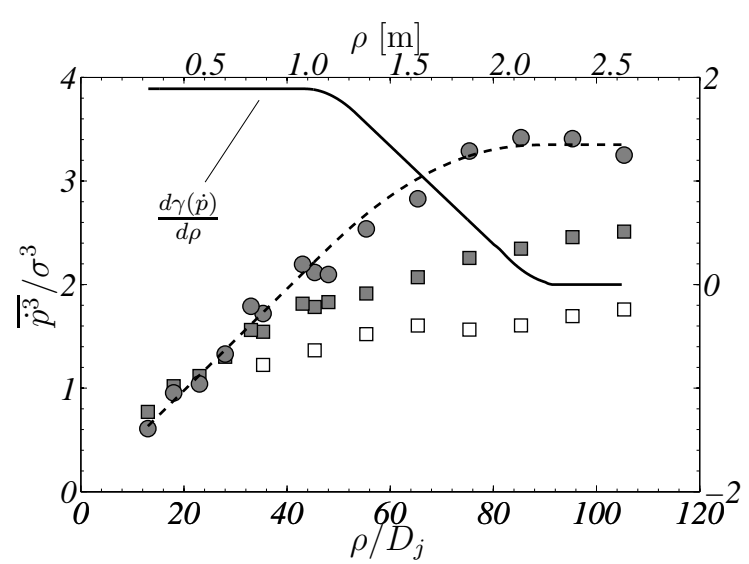

(b)

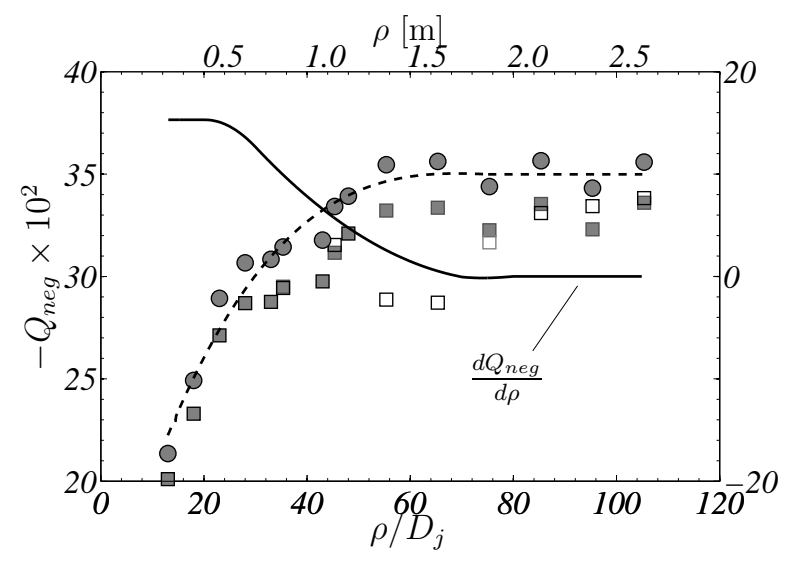

(d)

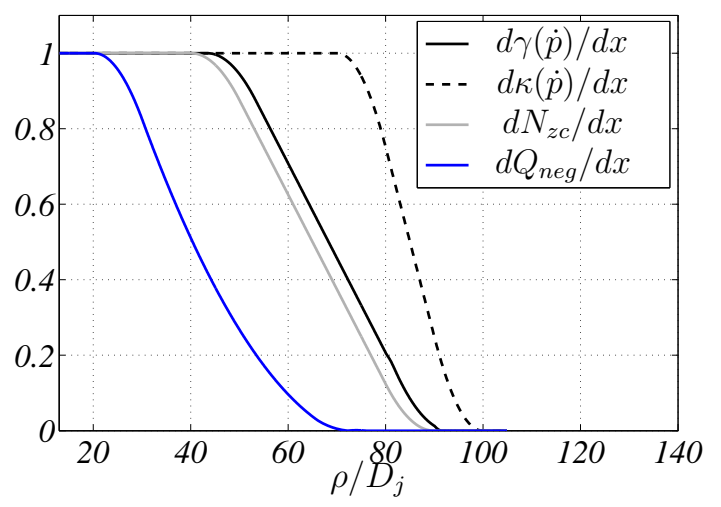

(e)

Figure 4.3: (a) $N_{z c}$, (b) $\gamma(\dot{p})$, (c) $\kappa(\dot{p})$, (d) $-Q_{\text {neg }} \times 10^{2}$ along the $45^{\circ}$ ray and (e) comparison of their normalized spatial derivatives. 


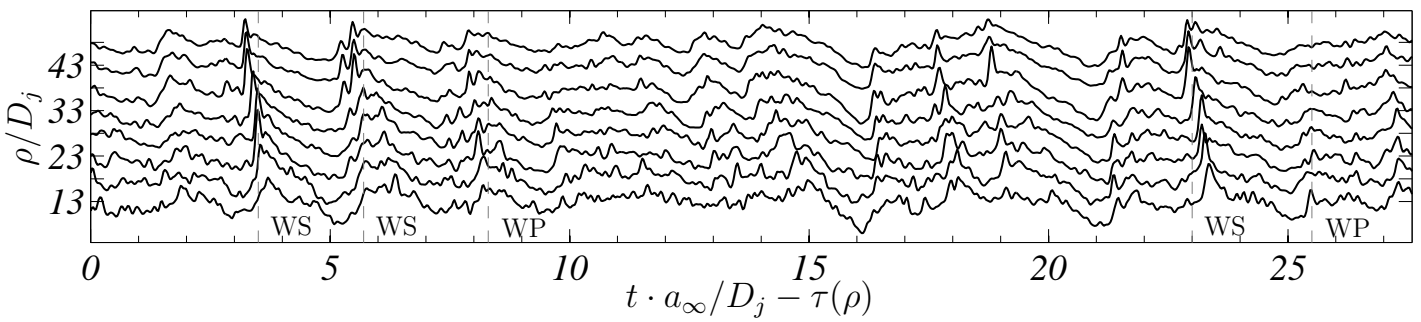

Figure 4.4: Evolution of the raw pressure time series (shown as $p(\rho, t) / \sigma_{p}(\rho)$ ) along the $45_{1}^{\circ}$ ray in the inner zone encompassing $\rho / D_{j}=[13: 5: 48]$.

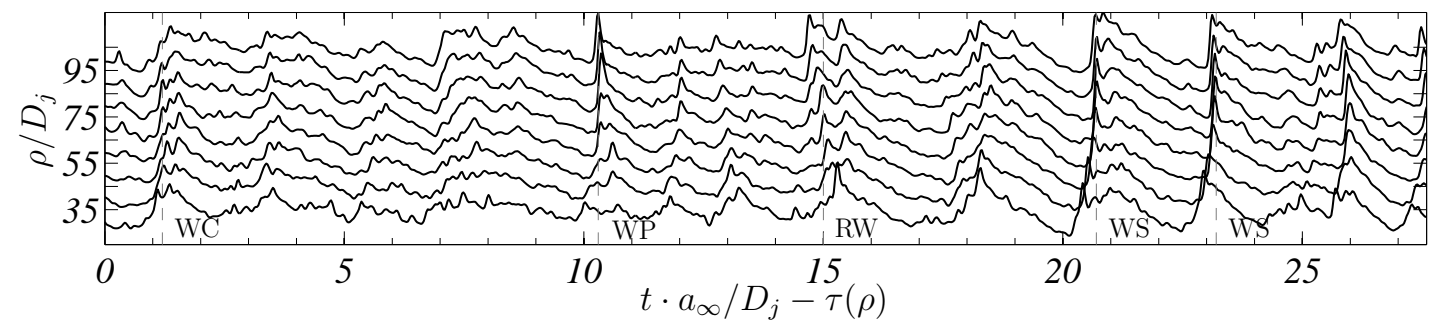

Figure 4.5: Evolution of the raw pressure time series (shown as $p(\rho, t) / \sigma_{p}(\rho)$ ) along the $45_{2}^{\circ}$ ray in the outer zone encompassing $\rho / D_{j}=[35.5: 10: 105.5]$.

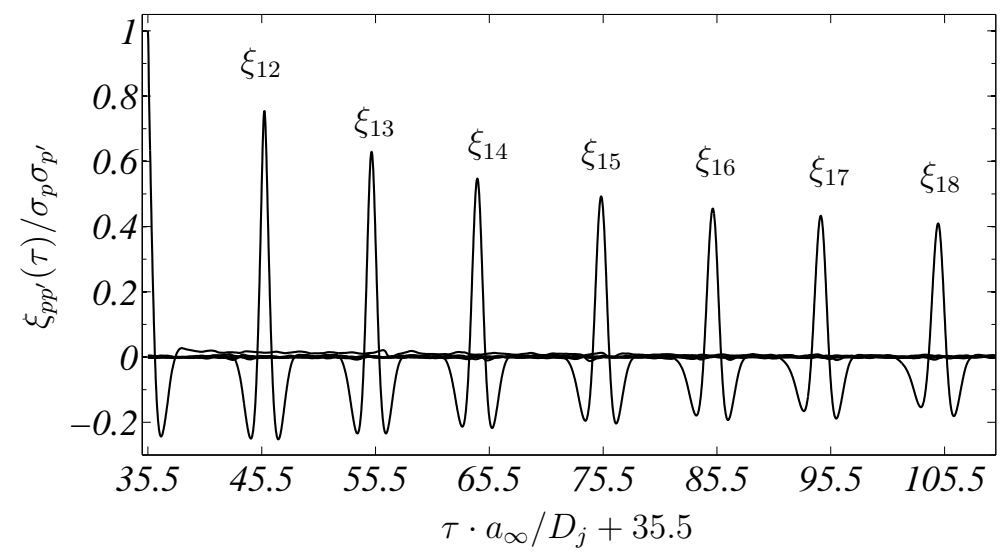

Figure 4.6: Cross-correlations between microphones on the $45_{2}^{\circ}$ ray in the outer zone encompassing $\rho / D_{j}=[35.5: 10: 105.5]$. 


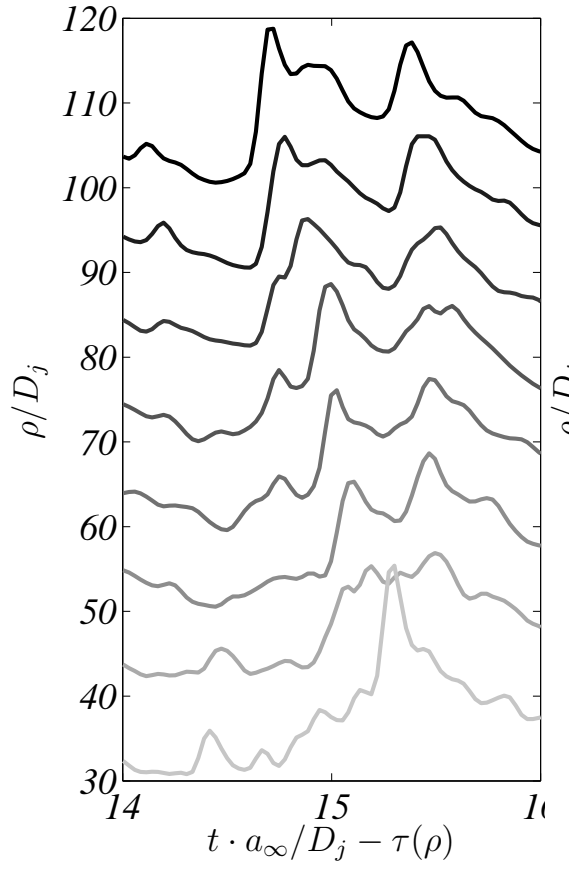

(a)

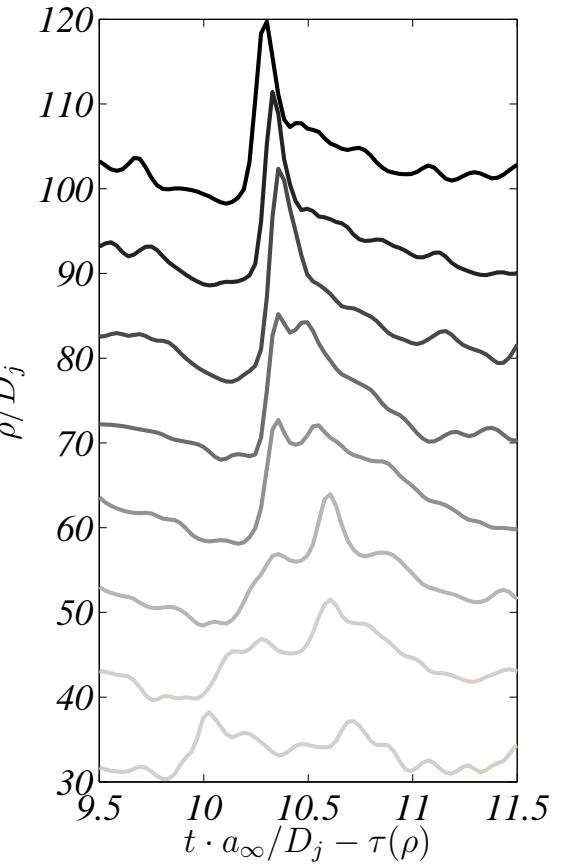

(b)

Figure 4.7: (a) A rogue wave and (b) a passing wave. 


\section{Chapter 5}

\section{Conclusions}

The acoustic waveforms produced by an unheated Mach 3 jet are investigated using $1 / 4$ inch microphones covering a 2 -D grid in the $(x, r)$ plane as well as 1/8 inch microphones placed along rays emanating from the source regions of the flow at $x / D_{j}=17.5$. Various metrics including pressure amplitude decay, the normalized quadrature spectral density, skewness of the

pressure derivative, the number of zero crossings and the integral of the negative part of the quadrature spectral density were used to study the behavior of these waveforms along the propagation path. Where the pressure amplitude is concerned, the location where its rate of decay first abides by cylindrical spreading, followed by spherical spreading, was identified and was confirmed using a simple wave packet model. The OASPL appeared not to be affected by sensor size and so the linear prediction of its amplitude at 95 jet diameters was performed and shown to collapse remarkably well for all azimuthal angles when propagated along rays emanating from the post potential core regions of the jet.

Both spectral and scalar metrics used to compute nonlinear waveform distortions revealed three different regions in the jet near-field. The first comprised a zone where waveform distortion appears to be increasing at a constant rate that initiates in close proximity to the jet (within one meter from the prominent source region). A second zone showed a decrease in nonlinear 
distortion effects while a third zone demonstrated how the waveforms were no longer steepening and had either reached their maximum or were beginning to undergo a state of relaxation. A visual extrapolation of the nonlinear metrics towards the source region suggests that the acoustic waveforms are initially distorted at the source. Consequently, a direct observation of the raw acoustic time series along the propagation path revealed both passing waves and rogue waves in the pressure waveform, both of which would ultimately increases the metrics for quantifying cumulative nonlinear distortion. 


\section{Bibliography}

[1] Anderson, A. T. and Freund, J.B., "Source mechanisms of jet crackle," 18th AIAA/CEAS Aeroacoustics Conference, AIAA Paper 2012-2251, 2012.

[2] Baars, W. J., Tinney, C. E. and Wochner, M.S., "Nonlinear Propagation of Sound from a Fully Expanded Mach 3 Jet," 50th AIAA Aerospace Sciences Meeting, AIAA Paper 2012-1177, 2012.

[3] Baars, W. J., "Acoustics from high-speed jets with crackle," PhD thesis, The University of Texas at Austin, May 2013.

[4] Baars, W.J. and Tinney, C.E., "Shock-structures in the acoustic field of a Mach 3 jet with crackle," Journal of Sound and Vibration, 2014, in publication, pp. 1-15.

[5] Baars, W.J., Tinney, C.E., Wochner, M. and Hamilton, M.F., "On cumulative nonlinear acoustic waveform distortions from high-speed jets," Journal of Fluid Mechanics, Vol. 749, 2014, pp. 331-366.

[6] Bass, H.E., Sutherland, L.C., Zucherwar, A.J., Blackstock, D.T. and Hester, D.M., "Atmospheric absorption in air: Further developments" Journal of the Acoustical Society of America, Vol. 97, 1996, pp. 680-683.

[7] Blackstock, D.T., "Fundamentals of physical acoustics," John Wiley and Sons, Inc, New York, NY, 2000, pp. 513-518. 
[8] Donald, B.W., Baars, W. J., Tinney, C. E. and Ruf, J.H., "Sound produced by large area ratio nozzles during fixed and transient operations," $A I A A$ Journal, DOI:10.2514/1.J052588, pp. 1-12.

[9] Ffowcs Williams, J.E., and Maidanik, G., "The mach wave field radiated by supersonic turbulent shear flows," Journal of Fluid Mechanics, Vol. 21, 1965, pp. 641-657.

[10] Ffowcs Williams, J.E., Simson, J. and Virchis, V.J., "Crackle': an annoying component of jet noise," Journal of Fluid Mechanics, Vol. 71, No. 2, 1975, pp. 251-271.

[11] Freund, J.B., "Noise sources in a low-Reynolds-number turbulent jet at Mach 0.9" Journal of Fluid Mechanics, Vol. 438, 2001, pp. 277-305.

[12] Gee, K.L., Sparrow, V.W., James, M.M., Downing, J.K., Hobbs, C.M., Gabrielson, T.B. and Atchley, A.A., "The role of nonlinear effects in the propagation of noise from high-power jet aircraft," Journal of the Acoustical Society of America, Vol. 123, No. 6, 2008, pp. 4082-4092.

[13] Goldstein, M.E., "On identifying the true sources of aerodynamic sound," Journal of Fluid Mechanics Vol. 526, 2005, pp. 337-347.

[14] Grizzi, S. and Camussi, R., "Wavelet analysis of near-field pressure fluctuations generated by a subsonic jet," Journal of Fluid Mechanics, Vol. 698, 2012, pp. 93-124.

[15] Hamilton, M.F. and Blackstock, D.T., "Nonlinear Acoustics," Acoustical Society of America, chap. 4, 2008. 
[16] Hamilton, M.F., "Effective Gol'dberg number for diverging waves" 166th Meeting of the Acoustical Society of America, Vol. 134, No. 5, Pt. 2 of 2. 2013, pp. 680-683.

[17] Howell, G.P. and Morfey, C.L., "Non-linear propagation of broadband noise signals," Journal of Sound and Vibration, Vol. 114, No. 2, 1987, pp. 189-201.

[18] James, M., Salton, A., Gee, K., Neilson, T., McInerny, S. and Kenny, R., "Modification of directivity curves for a rocket noise model," Proceedings from the Acoustical Society of America", Vol. 18, Session 3aNS: Noise, 2012.

[19] Jordan, P. and Colonius, T., "Wave packets and turbulent jet noise," Annu. Rev. Fluid Mech., Vol. 45, 2013, pp. 173-195.

[20] Kuo, C.-W., Veltin, J. and McLaughlin, D.K., "Effect of jet source distribution on acoustic far-field measurements," International Journal of Aeroacoustics, Vol. 11, No. 7-8, 2012, pp. 885-915.

[21] Kuo, C., Du, Y., McLaughlin, D.K. and Morris, P.J., "Experimental and computational study of near field / far field correlations in supersonic jet noise," 50th Aerospace Sciences Meeting, AIAA Paper 2012-1170, 2012.

[22] Krothapalli, A. and Arakeri, V., "Mach wave radiation : a review and an extension," AIAA Paper 2003-1200, 2003.

[23] Lighthill, M.J., "On sound generated aerodynamically: I. General Theory," Proc. R. Soc. Lond. A, Vol. 211, 1952, pp. 564-587. 
[24] Lilley, G.M., "On the noise from jets," Noise Mechanisms AGARD-CP131, 1974, pp. 13.1-13.12.

[25] Mayes, W.H., Lanford, W.E. and Hubbard, H.H., "Near-field and far-field noise surveys of solid-fuel rocket engines for a range of nozzle exit pressures," NASA TN D-21, August, 1959.

[26] McInerny, S.A., "Launch vehicle acoustics part 2 : statistics of the time domain data," Journal Aircraft, Vol. 33, No. 3, 1996, pp. 512-523.

[27] McLaughlin, D.K., Morrison, G.L. and Troutt, T.R., "Experiments of the instability waves in supersonic jet and their acoustic radiation," Journal of Fluid Mechanics, Vol. 69, No. 1, 1975, pp. 73-95.

[28] Morfey, C.L. and Howell, G.P., "Nonlinear propagation of aircraft noise in the atmosphere," AIAA Journal, Vol. 19, No. 8, 1981, pp. 986-992.

[29] Morris, P.J., "A note on noise generation by large scale structures in subsonic and supersonic jets," International Journal of Aeroacoustics, Vol. 8, No. 4, 2009, pp. 301-316.

[30] Nichols, J.W., Lele, S.K., Ham, F.E., Martens, S. and Spyropoulos, J.T.," Crackle Noise in Heated Supersonic Jets"," Journal of Engineering for Gas Turbines and Power, Vol. 135, 2013.

[31] Papamoschou, D., "Wavepacket modeling of the jet noise source," 17th AIAA/CEAS Aeroacoustics Conference, AIAA Paper 2011-2835, Portland, Or, 2011. 
[32] Petitjean, B., McLaughlin and D.K., "Experiments on the nonlinear propagation of noise from supersonic jets," 9th AIAA/CEAS Aeroacoustics Conference, AIAA Paper 2003-3127, 2003.

[33] Potter, R.C. and Jones, J.H., "An experiment to locate the acoustic sources in a high speed jet exhaust stream," Wyle Laboratories Report, 1967.

[34] Tam, C. K. W. and Chen, P., "Turbulent mixing noise from supersonic jets," AIAA Journal, Vol. 32, No. 9, 1994, pp. 1774-1780.

[35] Tam, C.K.W., Golebiowski, M. and Seiner, J.M., "On the two components of turbulent mixing noise from supersonics jets," 2nd AIAA/CEAS Aeroacoustics Conference, AIAA Paper 1996-1716, 1996.

[36] Tam, C. K. W., "Mach wave radiation from high-speed jets," AIAA Journal, Vol. 47, No. 10, 2009, pp. 2440-2448.

[37] Tinney, C.E. and Jordan, P., "The near pressure field of co-axial subsonic jets," Journal of Fluid Mechanics, Vol. 611, 2008, pp. 175-204.

[38] Tinney, C.E. Glauser, M.N., and Ukeiley, L.S., "Low-dimensional characteristics of a transonic jet. Part 1: Proper orthogonal decomposition," Journal of Fluid Mechanics, Vol. 612, 2008, pp. 107-141. 